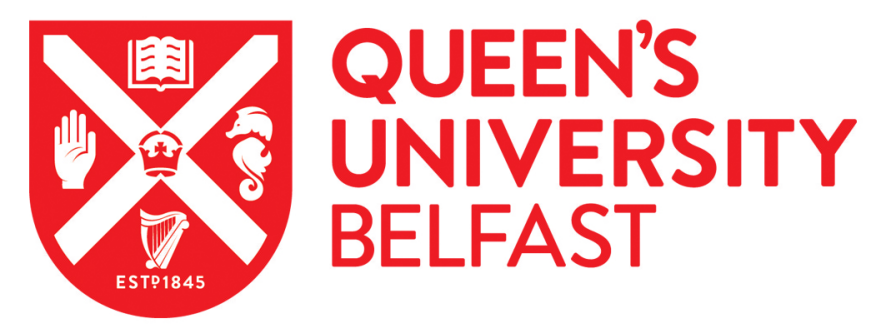

\title{
Testing of a Market Fraction Model and Power-law Behaviour in the DAX 30
}

He, X-Z., \& Li, Y. (2015). Testing of a Market Fraction Model and Power-law Behaviour in the DAX 30. Journal of Empirical Finance, 31,1-17. https://doi.org/10.1016/j.jempfin.2015.01.001

Published in:

Journal of Empirical Finance

Document Version:

Peer reviewed version

Queen's University Belfast - Research Portal:

Link to publication record in Queen's University Belfast Research Portal

Publisher rights

Copyright 2015 Elsevier

This is an open access article published under a Creative Commons Attribution-NonCommercial-NoDerivs License

(https://creativecommons.org/licenses/by-nc-nd/4.0/), which permits distribution and reproduction for non-commercial purposes, provided the author and source are cited.

\section{General rights}

Copyright for the publications made accessible via the Queen's University Belfast Research Portal is retained by the author(s) and / or other copyright owners and it is a condition of accessing these publications that users recognise and abide by the legal requirements associated with these rights.

\section{Take down policy}

The Research Portal is Queen's institutional repository that provides access to Queen's research output. Every effort has been made to ensure that content in the Research Portal does not infringe any person's rights, or applicable UK laws. If you discover content in the Research Portal that you believe breaches copyright or violates any law, please contact openaccess@qub.ac.uk. 


\title{
TESTING OF A MARKET FRACTION MODEL AND POWER-LAW BEHAVIOUR IN THE DAX 30
}

\author{
XUE-ZHONG HE* AND YOUWEI LI** \\ *UTS Business School \\ University of Technology, Sydney \\ PO Box 123 Broadway \\ NSW 2007, Australia \\ Tony.He-1@uts. edu . au \\ and \\ **School of Management \\ Queen's University of Belfast
}

Riddel Hall, 185 Stranmillis Road

BT9 5EE, Belfast, UK

y.li@qub.ac.uk

Date: Latest version: January 21, 2015.

Acknowledgements: This research was initiated and conducted during He's visit at Queen's University Belfast and Li's visit to Quantitative Finance Research Center at University of Technology, Sydney, whose hospitality they gratefully acknowledge. Financial support from the Australian Research Council (ARC) under discovery grant (DP130103210) is also gratefully acknowledged. We benefited from detailed comments of the anonymous referee, Professor Michael Goldstein of Babson College, and Professor Bertrand Melenberg of Tilburg University on the earlier version of this paper. The authors also would like to thank Colin O'Hare for the careful reading of the paper. The usual caveat applies.

Corresponding author: Youwei Li, School of Management, Queen's University of Belfast, Riddel Hall, 185 Stranmillis Road, BT9 5EE, Belfast, United Kingdom. Email: y.li@qub.ac.uk. Ph: (44 28) 90974826. Fax: (44 28) 90974201. 
ABSTRACT. This paper tests a simple market fraction asset pricing model with heterogeneous agents. By selecting a set of structural parameters of the model through a systematic procedure, we show that the autocorrelations (of returns, absolute returns and squared returns) of the market fraction model share the same pattern as those of the DAX 30. By conducting econometric analysis via Monte Carlo simulations, we characterize these power-law behaviours and find that estimates of the power-law decay indices, the (FI)GARCH parameters, and the tail index of the selected market fraction model closely match those of the DAX 30. The results strongly support the explanatory power of the heterogeneous agent models.

\section{JEL Classification: C15, D84, G12}

Keywords and Phrases: Asset pricing, fundamentalists and trend followers, (FI)GARCH, powerlaw, tail index. 


\section{INTRODUCTION}

Traditional economic and finance theory is based on the assumptions of investor homogeneity and rational expectations. Since agents are rationally impounding all relevant information into their trading decisions, the movement of prices is assumed to be perfectly random and hence exhibit random walk behaviour. This view is the theoretical underpinning of the efficient market hypothesis and asset pricing theories generally either implicitly or explicitly, including the optimal portfolio rules developed by Markowitz (1952) and Merton (1971), the static and intertemporal capital asset pricing model of Sharpe (1964), Lintner (1965), Mossin (1966) and Merton (1973a) and models for the pricing of contingent claims beginning with the work of Black and Scholes (1973) and Merton (1973b). The impressive statistical evidence in favour of market efficiency, documented by Fama (1976), has been taken as support for the random walk model and for a long time financial economists were contented with this view as the explanation of the time series behaviour of observed asset prices.

Empirical investigations of (high-frequency) financial time series in both equity and foreign exchange markets show some common features that are not in line with these assumptions, so-called stylized facts: include excess volatility (relative to the dividends and underlying cash flows), excess skewness, fat tails (the tails of distribution have a higher density than that predicted by the normal distribution, which conventionally was indicated by excess kurtosis), volatility clustering (high/low fluctuations are followed by high/low fluctuations), and longrange dependence in volatility (often characterized by slow decay of autocorrelations of squared or absolute returns. More precisely, we see insignificant autocorrelations (ACs) of raw returns and hyperbolic decline of ACs of the absolute and squared returns, see Ding et al., 1993), and various power-law behaviour. We refer to Pagan (1996) for a comprehensive discussion of stylized facts characterizing financial time series and Lux (2008) for a recent survey on empirical evidence of various power laws. These facts are not entirely contradictory to the traditional economic and finance theory with representative agent and rational expectations, but the theory does not provide persuasive explanation on a large subset of these facts. Our paper contributes to the explanation of the stylized facts from a heterogeneous agent models (HAMs) perspective.

Our paper considers a simple market fraction (MF) model developed by $\mathrm{He}$ and Li (2007, 2008). The MF model is a simple stochastic asset pricing model, involving two types of traders 
(fundamentalists and trend followers) under a market maker scenario. He and Li (2008) describe various aspects of financial market behaviour and establish the connection between the stochastic model and its underlying deterministic system. Through a statistical analysis, $\mathrm{He}$ and $\mathrm{Li}$ (2008) show that convergence of market price to fundamental value, long- and short-run profitability of the two trading strategies, survivability of trend followers and various under- and over-reaction autocorrelation patterns of the stochastic model can be explained by the dynamics, including the stability and bifurcations, of the underlying deterministic system. Based on these results, $\mathrm{He}$ and $\mathrm{Li}$ (2007) study the generating mechanism of the MF model to produce the volatility clustering and the long-range dependence in volatility. The results show that heterogeneity, risk-adjusted trend chasing, and the interplay of a stable deterministic equilibrium and stochastic noisy processes can be the source of power-law distributed fluctuations. The power-law behaviour is further verified by econometric estimates via a Monte Carlo simulation. The analysis of generating mechanism and power-law decay estimation based on simulations in $\mathrm{He}$ and Li (2007) provides a promising perspective for testing of the MF model to actual data in this paper.

In this paper, we test the MF model using the daily DAX 30 index and pay particular attention on the power-law behaviour in volatility. By selecting a set of structural parameters of the model through a systematic procedure, we show that the autocorrelations of returns, absolute returns and squared returns of the MF model share the same patterns for the DAX 30 . By conducting econometric analysis via Monte Carlo simulations, we then characterize these power-law behaviours and find that estimates of the power-law decay indices, the (FI)GARCH parameters, and the tail index of the selected market fraction model match closely to the corresponding estimates for the DAX 30. Interpretation of the selected parameters is consistent with the power-law behaviour generating mechanism in $\mathrm{He}$ and $\mathrm{Li}$ (2007). The results provide very positive evidence on the explanatory power of heterogeneous agent models.

We should emphasize that econometric analysis, especially estimation of heterogeneous agent models is still a challenging task, see for instance, a recent comprehensive review by Chen et al. (2012). Generally, the difficulties of estimation come from the complexity of the HAMs, together with (typically) many parameters, which makes verification of identification rather difficult, and thus proving consistency of estimation troublesome. For recent attempts to estimate HAMs, the identification problem is typically circumvented by focussing on a relatively simple 
HAMs, or by estimating a few key parameters only. Boswijk et al. (2007) derive a reduced form equation from a simplified Brock and Hommes $(1997,1998)$ type model and estimate it by using nonlinear least square method. Alfarano et al. (2005) estimate a simplified herding model by maximum likelihood method. Amilon (2008) estimates two specifications of the extended Brock and Hommes switching models described in De Grauwe and Grimaldi (2003, 2006) by using the efficient method of moments and maximum likelihood method. He concludes that the simple prototype models he estimated seem to have potential to explain empirical facts although the fit is generally not quite satisfactory. But he also reports local minima, possibly not the global minimum, when calculating the estimators. Franke (2009) applies the method of simulated moments to a model developed by Manzan and Westerhoff (2005). He reports that one of the parameters is not identified. He also discusses the problem of many local minima. Nevertheless, the other (identified) parameters could be estimated in a meaningful way, despite the simplicity of the model. Thus, although good progress seems to be made in estimating HAMs, even in case consistent estimation would be possible, the likely heavily nonlinear relationship between observables and unknown parameters to be estimated might seriously complicate estimation, see, for example, Chen et al. (2012), and experienced by, for example, Amilon (2008) and Franke (2009). Therefore, in this paper, following Li et al. (2010) we select structural parameters of interests in the MF model class that minimizes a distance between data based and HAMs based parameters.

Our approach seems relevant to general HAMs, particularly when dealing with more complicated models. Moreover, quite possibly a HAM might be misspecified, so that likelihood and/or moments based methods might produce poor results. For instance, this might be the reason that in Franke (2009) the method of simulated moments, using the optimal weighting matrix, produces less favourable results compared to estimating the parameters based on an intuitive way of linking theoretical and empirical moments. Our procedure is more robust to model misspecification, sharing the same spirit as real business cycles literature, such as Kydland and Prescott (1982), and equity premium puzzle literature, Mehra and Prescott (1985), Diebold et al. (1998) and Schorfheide (2000) in the macroeconomics literature, Gilli and Winker (2003), and Winker and Gilli (2003) in agent-based financial market literature

${ }^{1}$ For the discussions on comparison of this methodology with the usual estimation methodology, see, e.g., Canova (1994), Hansen and Heckman (1996), Kydland and Prescott (1991, 1996), Geweke (2006), and Dridi et al. (2007). 
The remainder of the paper is organized as follows. Section 2 provides a selective literature review on the development of HAMs. Section 3 summaries the MF model. In Section 4 we first systematically select a set of structural parameters of the MF model to characterize the power-law behaviour in volatility of the DAX 30 stock market daily closing price index. We then estimate the power-law decay parameters of the autocorrelation of returns, the squared returns and the absolute returns, $(\mathrm{FI}) \mathrm{GARCH}(1,1)$ parameters, and the power-law decay rates of the tail distribution for both the DAX 30 index and MF model-generated data. In Section 5, we present an explanation of the selected structural parameters of the MF model. Section 6 concludes.

\section{LiterATURE REVIEW}

Various statistical models have been developed to characterize the stylized facts. For instance GARCH processes initiated by Engle (1982) model returns as a random process with a time-varying variance that shows autoregressive dependence. These models produce volatility clustering and fat tails of the unconditional distribution. However, the implied decay of the volatility autocorrelation is exponential rather than hyperbolic, which is in contradiction with the long-range dependence in volatility. Other popular statistical models include variations of the ARCH-class model and the Markov switching model, for instance, see Bollerslev et al. (1986) and Hamilton (1994). These models are quite successful in modelling some of these stylized facts, but they do not offer any economic explanation.

As a result, the literature has witnessed increased attempts at modelling the financial markets by incorporating heterogeneous agents and bounded rationality, see surveys by Hommes (2006), LeBaron (2006), Lux (2008, 2009), Chiarella et al. (2009) and Chen et al. (2012). These models characterize the dynamics of financial asset prices resulting from the interaction of heterogeneous agents having different attitudes to risk and having different expectations about the future evolution of prices. One of the key aspects of these models is that they exhibit feedback of expectations - the agents' decisions are based upon predictions of future values of endogenous variables whose actual values are determined by equilibrium equations. In particular, Brock and Hommes $(1997,1998)$ proposed an Adaptive Belief System model of economic and financial markets. The agents adapt their beliefs over time by choosing from different predictors or 
expectation functions, based upon their past performance. The resulting nonlinear dynamical system is, as Brock and Hommes (1998) and Hommes (2002) show, capable of generating a wide range of complex price behaviour from local stability to high order cycles and chaos. It is very interesting to find that adaptation, evolution, heterogeneity, and even learning, can be incorporated into the Brock and Hommes type of framework2. Moreover, recent works by Westerhoff (2004), Chiarella et al. (2005) and Westerhoff and Dieci (2006) show that complex price dynamics may also result within a multi-asset market framework. This broader framework also gives rise to rich and complicated dynamics and can be used to obtain a deeper understanding of market behaviour. They are capable of explaining various market behaviours, such as the deviation of the market price from the fundamental price, market booming and crashes.

One of the most interesting questions for the above heterogeneous agent models is how well they can explain the stylized facts. In most of this literature so far, numerical simulations have shown that the models are able to generate some of the stylized facts of financial markets, including volatility clustering, excess skewness and kurtosis, and fat tails, but not all. In particular, explanation of the power-law behaviou1 3 still seems to be a challenging task.

This has spurred attempts at a theoretical explanation by using HAMs and the search for an understanding of the underlying mechanisms that are responsible for such power laws]. Multiplicative stochastic processes (with multiplicative and additive stochastic components) have been used to explain the power-law behaviour in rational bubble models (see Kesten (1973) and Lux (2008)). However, as shown by Lux and Sornette (2002), the range of the exponent required for the rational bubble models is very different from the exponent observed from the empirical findings. In addition, the rational bubble models share the conceptual problems of economic models with fully rational agents. Herding models of financial markets have been developed to incorporate herding and contagion phenomena 55 With a stripped down version of an extremely parsimonious stochastic herding model with fundamentalists (who trade on

\footnotetext{
${ }^{2}$ See, Hommes $(2001,2002)$, Chiarella and He (2002, 2003b), Chiarella et al. (2002), Westerhoff (2003) and De Grauwe and Grimaldi (2006).

${ }^{3}$ They include power-law distribution of large returns, hyperbolic decline of return autocorrelation function, temporal scaling of trading volume and multi-scaling of higher moments of returns.

${ }^{4}$ For instance, Farmer et al. (2004) attribute the power-law behaviour in return distribution to liquidity fluctuation using market microstructure approach. We refer to Lux (2008) for a recent survey on empirical evidence, models and mechanisms of various financial power laws.

${ }^{5}$ See Kirman (1991, 1993), Lux (1995, 1997, 1998), Lux and Marchesi (1999), Chen et al. (2001), and Aoki and Yoshikawa (2002). Lux and Marchesi (1999) argue that the indeterminateness of the market fractions in a market equilibrium and the dependence of stability on the market fractions exist in a broad class of behavioural finance models, which is further supported by Giardina and Bouchaud (2003) and Lux and Schornstein (2005).
} 
observed mispricing) and noise traders (who follow the mood of the market), Alfarano et al. (2005) show that their herding model is able to produce relatively realistic time series for returns whose distributional and temporal characteristics are astonishingly close to the empirical findings. This is partly due to a bi-modal limiting distribution for the fraction of noise traders in the optimistic and pessimistic groups of individuals and partly due to the stochastic nature of the process leading to recurrent switches from one majority to another. A mechanism of switching between predictors and co-existing attractors is used in Gaunersdorfer and Hommes (2007) to characterize volatility clustering 6 . The highly nonlinear deterministic system may exhibit coexistence of different types of attractors and adding noise to the deterministic system may then trigger switches between low- and high-volatility phases. Their numerical simulations show quite satisfactory statistics between the simulated and actual data. However, the comparison with empirical facts is mainly based upon visual inspection, or upon a few realizations of the model without formal econometric treatment.

In contrast to the theoretical oriented models discussed above, there is also a rapidly expanding literature of HAMs which are computationally oriented and we refer the reader to a recent survey by LeBaron (2006). One of the most important advantages of this approach is that many behavioural aspects at the micro level including the interaction of agents can be aggregated at the macro level through computer simulations. However, as pointed by Hommes (2006), HAMs, particularly the computational HAMs, face a problem of too many degrees of freedom and too many parameters, which makes it difficult to assess the main causes of observed stylized facts. This problem makes the estimation and calibration of the HAMs model to financial data difficult.

In terms of the comparison of the econometric characterizations between the simulation models and the actual data, we refer to, Chen et al. (2012) for an overview and Li et al. $(2006,2010)$ for a formal analysis. One of the important issues in dealing with the estimation of HAMs, as pointed out by Hommes (2006), is "to understand the generating mechanism of the stylized facts, one would like to find the simplest HAM with a plausible behavioural story at the micro level, that still captures the most important stylized facts observed at the aggregate level. $\cdots$.

\footnotetext{
${ }^{6}$ Other behavioural finance explanations for volatility clustering include Manzan and Westerhoff (2005) who develop a model in which traders tend to over- or under-react to the arrival of new information.
} 
Simple and parsimonious HAMs can thus help to discipline the wilderness of agent-based modelling". It is this principle that guides our approach of selecting structural parameters of the MF model to the DAX 30 in this paper.

\section{The Market Fraction Model with Heterogeneous Expectations}

The Market Fraction (MF) model considered in this section is a standard discounted value asset pricing model with heterogeneous agents. It is closely related to the framework of Brock and Hommes (1997, 1998) and Chiarella and He (2002). We outline the model and refer the readers to $\mathrm{He}$ and $\mathrm{Li}$ (2008) for full details.

Consider an economy with one risky asset, one risk free asset, and two types of traders with different beliefs or expectations on the future price of the risky asset. It is assumed that the risk free asset is perfectly elastic and supplied at gross return of $R=1+r / K$, where $r$ stands for a constant risk-free rate per annum and $K$ stands for the trading frequency measured in fractions of a year. $]^{7}$ Let $P_{t}$ and $D_{t}$ be the (ex dividend) price and dividend per share of the risky asset at time $t$, respectively. The first type of investor is called the fundamentalist (or informed trader) with a market fraction of population of $n_{1}$. The second type of investor is called the trend follower (or uninformed trader) with a market fraction of population of $n_{2}$. Note that $n_{1}+n_{2}=1$. Let $m=n_{1}-n_{2} \in[-1,1]$, then $m=1(-1)$ corresponds to the case when all the traders are the fundamentalists (trend followers).

For investor $i$, let $W_{i, t}$ be his/her initial wealth and $z_{i, t}$ be the number of shares of the risky asset held by the investor at $t$. Then the portfolio wealth of the investor $i$ at $t+1, W_{i, t+1}$, is given by

$$
W_{i, t+1}=R W_{i, t}+\left[P_{t+1}+D_{t+1}-R P_{t}\right] z_{i, t}
$$

Let $E_{h, t}$ and $V_{h, t}$ be the beliefs of type $h$ traders $(h=1,2)$ about the conditional expectation and variance of quantities at $t+1$ based on their information at time $t$. Denote by $R_{t+1}(=$ $\left.P_{t+1}+D_{t+1}-R P_{t}\right)$ the excess capital gain on the risky asset at $t+1$. Assume that trader $h$ has a constant absolute risk aversion (CARA) utility function with the risk aversion coefficient $a_{h}$ (e.g. $U_{h}(W)=-\exp \left(-a_{h} W\right)$ ). By expected utility maximization, the optimal demand on

\footnotetext{
${ }^{7}$ Typically, $K=1,12,52$ and 250 for trading period of year, month, week and day, respectively. To estimate the stylized facts observed from daily price movement in financial market, we select $K=250$ in our discussion.
} 
the risky asset of trader $h$ is given by

$$
z_{h, t}=\frac{E_{h, t}\left(R_{t+1}\right)}{a_{h} V_{h, t}\left(R_{t+1}\right)}, \quad h=1,2
$$

Assume zero supply of outside shares. Then, using (3.2), the population weighted aggregate excess demand $z_{e, t}$ is given by

$$
z_{e, t} \equiv n_{1} z_{1, t}+n_{2} z_{2, t}=\frac{1+m}{2} \frac{E_{1, t}\left[R_{t+1}\right]}{a_{1} V_{1, t}\left[R_{t+1}\right]}+\frac{1-m}{2} \frac{E_{2, t}\left[R_{t+1}\right]}{a_{2} V_{2, t}\left[R_{t+1}\right]} .
$$

We assume that the market price is determined by a market maker who cleans the market by taking a long (when $z_{e, t}<0$ ) or short (when $z_{e, t}>0$ ) position. At the end of period $t$, after the market maker has carried out all transactions, he or she adjusts the price for the next period in the direction of the observed excess demand with a speed of price adjustment of $\mu$. To capture unexpected market news or the excess demand of noise traders, we introduce a noisy demand term $\tilde{\delta}_{t}$ which is an i.i.d. normally distributed random variable with $\tilde{\delta}_{t} \sim \mathcal{N}\left(0, \sigma_{\delta}^{2}\right)$. Based on these assumptions and (3.3), the market price is determined by

$$
P_{t+1}=P_{t}+\frac{\mu}{2}\left[(1+m) \frac{E_{1, t}\left[R_{t+1}\right]}{a_{1} V_{1, t}\left[R_{t+1}\right]}+(1-m) \frac{E_{2, t}\left[R_{t+1}\right]}{a_{1} V_{2, t}\left[R_{t+1}\right]}\right]+\tilde{\delta}_{t} .
$$

We now turn to discuss the beliefs of fundamentalists and trend followers. Denote by $F_{t}=$ $\left\{P_{t}, P_{t-1}, \cdots ; D_{t}, D_{t-1}, \cdots\right\}$ the common information set formed at time $t$. We assume that, apart from the common information set, the fundamentalists have superior information on the fundamental value, $P_{t}^{*}$, of the risky asset, which is assumed to follow a stationary process 8

$$
P_{t+1}^{*}=P_{t}^{*} \exp \left(-\frac{\sigma_{\epsilon}^{2}}{2}+\sigma_{\epsilon} \tilde{\epsilon}_{t}\right), \quad \tilde{\epsilon}_{t} \sim \mathcal{N}(0,1), \quad \sigma_{\epsilon} \geq 0, \quad P_{0}^{*}=\bar{P}>0,
$$

where $\tilde{\epsilon}_{t}$ is independent of the noisy demand process $\tilde{\delta}_{t}$. This specification ensures that neither fat tails nor volatility clustering are brought about by the exogenous fundamental price process. Hence, any non-normal pattern in risky asset return, discussed below, would be driven by the trading process itself.

The fundamentalists are aware of trend followers and consequently believe that the stock price may be driven away from its fundamental value. More precisely, we assume that the

\footnotetext{
${ }^{8}$ The fundamental price process $P_{t}^{*}$ is an approximation of continuous log-normal price process with zero drift and volatility of $\sigma_{\epsilon}$. For a recent discussion on the specification of noise process in HAMs, see Franke (2010).
} 
conditional mean and variance of the fundamental traders are, respectively

$$
E_{1, t}\left(P_{t+1}\right)=P_{t}+\alpha\left(P_{t+1}^{*}-P_{t}\right), \quad V_{1, t}\left(P_{t+1}\right)=\sigma_{1}^{2}
$$

where $\sigma_{1}^{2}$ stands for a constant variance of the fundamental value, and $\alpha \in[0,1]$ is the fundamentalists' speed of price adjustment towards the fundamental value. In general, fundamental traders believe the market is efficient and prices converge to the expected fundamental value. A high (low) weight of $\alpha$ leads to a quick (slow) adjustment of expected price towards the fundamental price.

The trend followers, unlike the fundamental traders, are technical traders who believe the future price change can be predicted from various patterns or trends generated from the history of prices. The trend followers extrapolate the latest observed price change over a long-run sample mean of the history prices and to adjust their variance estimate accordingly. More precisely, their conditional mean and variance satisfy

$$
E_{2, t}\left(P_{t+1}\right)=P_{t}+\gamma\left(P_{t}-u_{t}\right), \quad V_{2, t}\left(P_{t+1}\right)=\sigma_{1}^{2}+b_{2} v_{t},
$$

where $\gamma, b_{2} \geq 0$ are constants, and $u_{t}$ and $v_{t}$ are the sample mean and variance, respectively, which may follow some learning processes. The parameter $\gamma$ measures the extrapolation rate and high (low) values of $\gamma$ correspond to strong (weak) extrapolation from the trend followers. The coefficient $b_{2}$ measures the influence of the sample variance. Various learning schemes 9 can be used to estimate the sample mean $u_{t}$ and variance $v_{t}$. Here we assume that:

$$
u_{t}=\delta u_{t-1}+(1-\delta) P_{t}, \quad v_{t}=\delta v_{t-1}+\delta(1-\delta)\left(P_{t}-u_{t-1}\right)^{2},
$$

where $\delta \in[0,1]$ is a constant. This is a limiting process of a geometric decay process when the length of memory lag tends to infinity 10 . The selection of this process is twofold. First, traders tend to put a high weight on the most recent prices and less weight on the more remote prices when they estimate the sample mean and variance. Second, the process leads to a low dimensional system which can be examined analytically. We note that instead of the geometric

\footnotetext{
${ }^{9}$ See for example Chiarella and $\mathrm{He}(2002,2003 a)$ for related studies.

${ }^{10}$ See Chiarella, He, Hung and Zhu (2006) for the proof. Basically, a geometric decay probability process $(1-\delta)\left\{1, \delta, \delta^{2}, \cdots\right\}$ is associated with the historical prices $\left\{P_{t}, P_{t-1}, P_{t-2}, \cdots\right\}$. The parameter $\delta$ measures the geometric decay rate. For $\delta=0$, the sample mean $u_{t}=P_{t}$, which is the latest observed price, while $\delta=0.1,0.5,0.95$ and 0.999 gives a half life of 0.43 day, 1 day, 2.5 weeks and 2.7 years, respectively.
} 
decay process, the trend following behaviour can also be modelled by other processes such as a moving average process in Chiarella, He, and Hommes (2006a, 2006b). However, with the moving average, the choice of the length of the moving window can become difficult. For different length of the moving window, the dimension of the resulted system is different and the analysis of the model has to be made separately. It also makes the analytical analysis of the underlying deterministic model more difficult, in particular when the length of the moving window is large. Empirically, we also have to estimate the length of the moving window as an extra parameter of the system. We should emphasize that it is the trend following behaviour, not a particular price trend process that plays more important role in explaining the power-law feature observed in real financial markets (see Chiarella, He and Hommes, 2006b). As explained in $\mathrm{He}$ and $\mathrm{Li}$ (2007), it is the interaction between the nonlinear deterministic dynamics and the noise processes that generates the power-law behaviour of the system. 11

To simplify the calculations, we assume that the dividend process $D_{t}$ follows $D_{t} \sim \mathcal{N}\left(\bar{D}, \sigma_{D}^{2}\right)$, the expected long-run fundamental value $\bar{P}=\bar{D} /(R-1)$, and the unconditional variances of the price $\left(\sigma_{1}^{2}\right)$ and dividend $\left(\sigma_{D}^{2}\right)$ over the trading period are related 12 by $\sigma_{D}^{2}=q \sigma_{1}^{2}$. Based on (3.6), $E_{1, t}\left(R_{t+1}\right)=\alpha\left(P_{t+1}^{*}-P_{t}\right)-(R-1)\left(P_{t}-\bar{P}\right), V_{1, t}\left(R_{t+1}\right)=(1+q) \sigma_{1}^{2}$ and hence the optimal demand of the fundamentalist is given by

$$
z_{1, t}=\frac{1}{a_{1}(1+q) \sigma_{1}^{2}}\left[\alpha\left(P_{t+1}^{*}-P_{t}\right)-(R-1)\left(P_{t}-\bar{P}\right)\right]
$$

Similarly, from (3.7), $E_{2, t}\left(R_{t+1}\right)=P_{t}+\gamma\left(P_{t}-u_{t}\right)+\bar{D}-R P_{t}=\gamma\left(P_{t}-u_{t}\right)-(R-1)\left(P_{t}-\right.$ $\bar{P}), V_{2, t}\left(R_{t+1}\right)=\sigma_{1}^{2}\left(1+q+b v_{t}\right)$, where $b=b_{2} / \sigma_{1}^{2}$. Hence the optimal demand of the trend follower is given by

$$
z_{2, t}=\frac{\gamma\left(P_{t}-u_{t}\right)-(R-1)\left(P_{t}-\bar{P}\right)}{a_{2} \sigma_{1}^{2}\left(1+q+b v_{t}\right)}
$$

\footnotetext{
${ }^{11}$ We thank the referee to point this out. We discuss this in details in Section 5 .

${ }^{12}$ Let $\sigma_{\bar{P}}$ be the annual volatility of $P_{t}^{*}$ and $\bar{D}_{t}=r P_{t}^{*}$ be the annual dividend. In this paper, we choose $\sigma_{1}^{2}=$ $\sigma_{\bar{P}}^{2} / K$ and $q=r^{2}$. In fact, the annual variance of the dividend $\bar{\sigma}_{D}^{2}=r^{2} \sigma_{\bar{P}}^{2}$. Therefore $\sigma_{D}^{2}=\bar{\sigma}_{D}^{2} / K=r^{2} \sigma_{\bar{P}}^{2} / K=$ $r^{2} \sigma_{1}^{2}$. In this paper, we choose $r=5 \%$ p.a, $\bar{P}=\$ 100, \sigma_{\bar{P}}=\sigma \bar{P}, \sigma_{\epsilon}=\sigma$ and $K=250$.
} 
Subsisting (3.9) and (3.10) into (3.4), the market price under a market maker is determined by the following 4-dimensional stochastic difference system

$$
\left\{\begin{aligned}
P_{t+1}= & P_{t}+\frac{\mu}{2}\left[\frac{1+m}{a_{1}(1+q) \sigma_{1}^{2}}\left[\alpha\left(P_{t+1}^{*}-P_{t}\right)-(R-1)\left(P_{t}-\bar{P}\right)\right]\right. \\
& \left.\quad+(1-m) \frac{\gamma\left(P_{t}-u_{t}\right)-(R-1)\left(P_{t}-\bar{P}\right)}{a_{2} \sigma_{1}^{2}\left(1+q+b v_{t}\right)}\right]+\tilde{\delta}_{t}, \\
u_{t}= & \delta u_{t-1}+(1-\delta) P_{t}, \\
v_{t}= & \delta v_{t-1}+\delta(1-\delta)\left(P_{t}-u_{t-1}\right)^{2}, \\
P_{t+1}^{*}= & P_{t}^{*} \exp \left(-\frac{\sigma_{\epsilon}^{2}}{2}+\sigma_{\epsilon} \tilde{\epsilon}_{t}\right) .
\end{aligned}\right.
$$

By applying the stability and bifurcation theory to the corresponding deterministic model and using Monte Carlo simulation to the stochastic model, He and Li (2008) conduct both analytical and statistical analysis for the model and find that the convergence of the market prices to their fundamental value, and various under and over-reaction autocorrelation patterns of returns can be characterized by the dynamics, including the stability and bifurcations, of the underlying deterministic system. Based on these characterizations, He and Li (2007) provide further evidence of the MF model on generating power-law behaviour in volatility, showing that agent heterogeneity, risk-adjusted trend chasing through the geometric learning process, and the interplay of noisy fundamental and demand processes and a stable deterministic equilibrium can be the source of power-law distributed fluctuations. In particular, the two noisy processes play different roles; the noisy demand plays an important role in the generation of insignificant ACs on returns, while the significant decaying AC patterns of the absolute returns and squared returns are more influenced by the noisy fundamental process. These findings provide a solid foundation for a systematic selection of structural parameters of the model to characterize financial data in terms of the power-law behaviour in volatility. In the following sections, we first systematically select the MF model to characterize the power-law behaviour of the DAX 30 and estimate the decay indices and (FI)GARCH parameters for the selected model. We then use the selected parameters to explain the market behaviour and provide further supporting evidence on the generating mechanism discovered in $\mathrm{He}$ and $\mathrm{Li}$ (2007). In addition, we reveal the potential of the MF model to characterize the power-law behaviour of tail distribution of asset returns. 


\section{Testing of the Power-Law Behaviour in the DAX 30}

This section illustrates how to systematically select structural parameters of the MF model to characterize the power-law behaviour of the DAX 30. We start with a brief discussion of the stylized facts of the DAX 30, including both fat tail and power-law behaviour. We then discuss the selection procedure, which is designed in principle to match the autocorrelation patterns in the returns, absolute and squared returns for the DAX 30, and present the selection result. Based on the selected parameters for the MF model, we use Monte Carlo simulations to examine the effectiveness of the selection procedure, estimate the decay indices of the power-law behaviour, and compare them with those of the DAX 30. In addition, we also examine the power-law tail behaviour of the selected MF model comparing it with the DAX 30. We demonstrate that the selected MF model generates closely the characterization of the power-law behaviour of the DAX 30 in the return autocorrelation and tails.

4.1. Stylized Facts and Autocorrelations of Returns for the DAX 30. The price index data for the DAX 30 comes from Datastream, which contains 8001 daily observations from 11 August, 1975 to 29 June, 2007. Use $p_{t}$ to denote the price index for the DAX 30 at time $t$ $(t=0, \ldots, 8000)$ and $\log$ returns $r_{t}$ are defined as $r_{t}=\ln p_{t}-\ln p_{t-1}(t=1, \cdots, 8000)$. Table 4.1 gives the summary statistics of $r_{t}$ for the DAX 30, which shows many stylized facts in financial markets. We can see from Table 4.1 that the kurtosis for $r_{t}$ is much higher than that of a normal distribution (which is 3 ). The kurtosis and studentized range statistics (which is the range divided by the standard deviation) show the characteristic fat-tailed behaviour compared with a normal distribution. The Jarque-Bera normality test statistic is far beyond the critical value, which suggests that $r_{t}$ is far from a normal distribution. Figures 4.1 (a) and (b) gives the plots of $p_{t}$ and $r_{t}$; they show that the market volatility is changing over time and large absolute returns are more likely to be followed by large absolute returns than small absolute returns. This suggests that a suitable model for the data should have a time varying volatility and volatility clustering structure as suggested by the ARCH and (FI)GARCH models.

TABLE 4.1. Summary statistics of $r_{t}$.

\begin{tabular}{cccccccc}
\hline mean & std. & skewness & kurtosis & $\min$ & $\max$ & stud. range & Jarque-Bera \\
\hline 0.00034 & 0.01244 & -0.4765 & 10.436 & -0.1371 & 0.0755 & 17.092 & 18735 \\
\hline
\end{tabular}



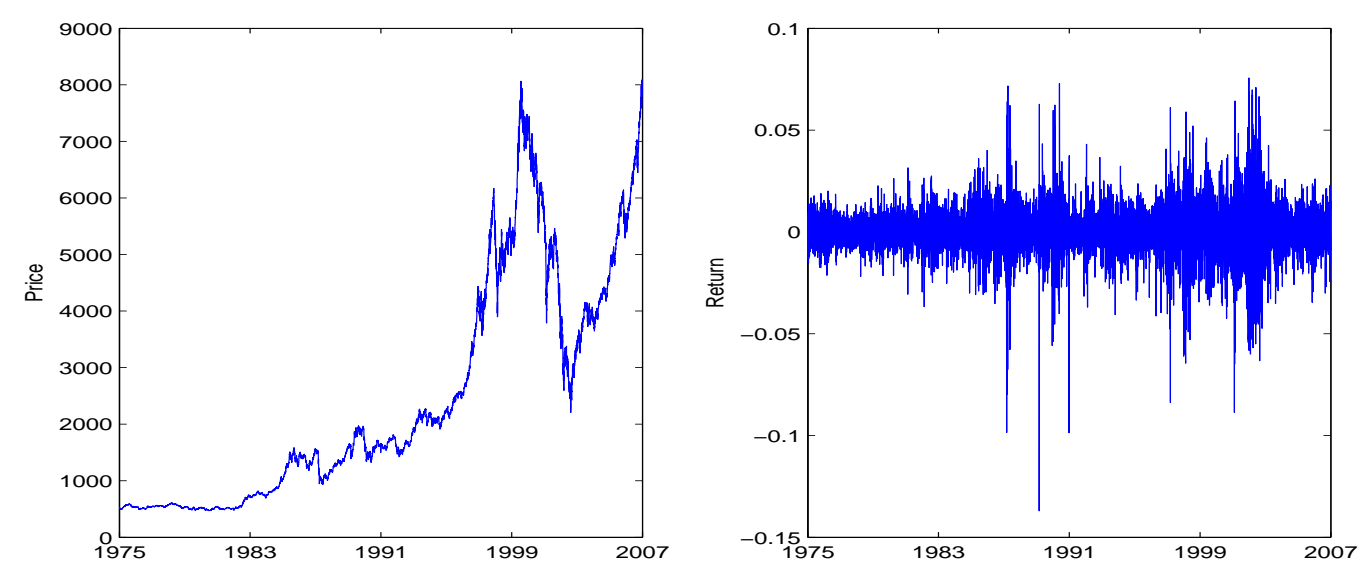

FIGURE 4.1. Time series on prices and log returns of the DAX 30 from 11 August, 1975 to 29 June, 2007.

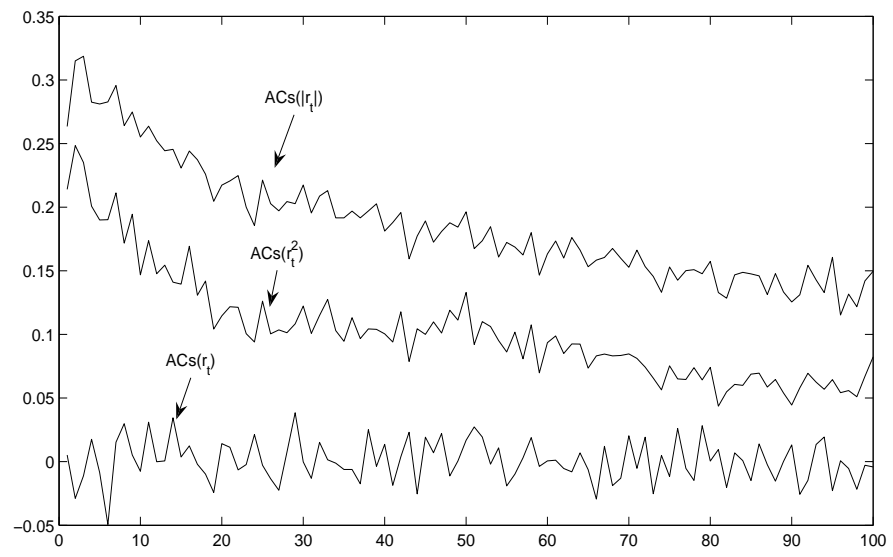

FIGURE 4.2. Autocorrelations of $r_{t}, r_{t}^{2}$ and $\left|r_{t}\right|$ for the DAX 30.

Apart from those reported stylized facts shared among different market indices, a well known stylized fact of stock returns is that the returns themselves contain little serial correlation, but the absolute return $\left|r_{t}\right|$ and the squared returns $r_{t}^{2}$ do have significantly positive serial correlations over long lags. For example, Ding et al. (1993) investigate ACs of returns (and their transformations) of the daily S\&P 500 index over the period 1928 to 1991 and find that the absolute returns and the squared returns tend to have very slow decaying autocorrelations, and further, the sample autocorrelations for the absolute returns were greater than those for the squared returns at every lag up to at least 100 lags. This kind of $\mathrm{AC}$ feature indicates the long-range dependence or the power-law behaviour in volatility. The autocorrelations for the DAX 30 are plotted in Figure 4.2, which clearly support the findings in Ding et al. (1993). 
4.2. Selection Method and Result. In principle, to select a set of structural parameters of the MF model matching the power-law behaviour of the DAX 30, we minimize the average distance between the autocorrelations of the log returns (the squared log returns, the absolute log returns) of the DAX 30 and the corresponding autocorrelations generated from the MF-models. More precisely, denote $\Theta$ the parameter space of the MF model. Let $\theta \in \Theta$ be the vector of parameters in the MF model that are of interest, $N$ be the number of independent simulations of the MF model, $\widehat{\beta}_{M F}^{n}$ be the estimated autocorrelations of the $n$-th run of the MF model, and $\widehat{\beta}_{D A X}$ be that of the DAX 30. In selection, we solve:

$$
\widehat{\theta} \in \arg \min _{\theta \in \Theta}\left\|\frac{1}{N} \sum_{n=1}^{N} \widehat{\beta}_{M F}^{n}-\widehat{\beta}_{D A X}\right\|^{2},
$$

for the standard Euclidian norm $\|\cdot\|$, using a generalized simplex algorithm. The parameters in the MF model are chosen to lie in the following ranges: $\alpha \in[0,1], \gamma \in[0.05,12], a_{1}, a_{2} \in$ $[0.001,8.0], \mu \in[0.1,5], m \in[-1,1], \delta \in[0,1], b \in[0.05,8.5], \sigma_{\varepsilon} \in[0.005,0.05], \sigma=\sqrt{K} \sigma_{\varepsilon}$ and $\sigma_{\delta} \in[0.05,8.5]$. However $\bar{P}=100$ and $q=r^{2}=0.05^{2}$ are kept fixed. In the selection and the subsequent econometric analysis, we ran 1,000 independent simulations over 9,000 time periods and discarded the first 1,000 time periods to wash out the possible initial noise effect. For each run of the model we obtain 8,000 observations to match the sample size of the DAX 30. It is not possible to use autocorrelations at all lags, so we focus on a limited set of autocorrelations. In particular, we focus on lag lengths of 1 to 50 , then $55,60,65, \ldots$, and up to 100 periods 13 . This corresponds to 60 autocorrelations in total for the return, absolute return and squared return, respectively. Essentially, the dimension of $\widehat{\beta}_{M F}^{n}$ and $\widehat{\beta}_{D A X}$ is 180 , with 60 autocorrelations estimated for each of the $r_{t}, r_{t}^{2}$ and $\left|r_{t}\right|$. The selected parameters of the MF model are reported in Table 4.2

We note that HAMs are highly likely to be misspecified, the selection procedure in (4.1) is based on the distance between the model and real world for a selected set of moments. It is designed to answer the question "given that the model is false, how true it is?" It allows us to focus on the characteristics in the data (in our case, this refers to the power law behaviour

\footnotetext{
${ }^{13} \mathrm{We}$ choose a large numbers of lags of ACs because our method of selection of the model parameters is exclusively focused on the ACs, and it works well to produce reasonable results reported in Figure 4.3. Note that some of the moments might be weak, and we might have too many moments (see; e.g., Newey and Windmeijier, 2009), the second order autocorrelations might be unstable in an estimation sense (see; e.g., Francq and Zakoian, 2000), and we also do not consider how to optimally select this set (Donald et al., 2008), their impact on the selection procedure need to be investigated further. We thank an anonymous referee to bring these potential issues to our attention.
} 
in volatility) for which the model is most relevant. A related important question is "to find out how wrong a model is and to compare the performance of different models" (Kan and Robotti, 2008, 2009). In representative agent and rational expectation setting, measures of model misspecification developed by Hansen and Jagannathan (1997) and recently, Kan and Robotti $(2008,2009)$ are used to rank model performance. The distance in (4.1) is an analogue of Hansen and Jagannathan measure of model misspecification in the context of HAMs. 14

We also note that (4.1) is analogous to the indirect estimator suggested by Gourieroux et al. (1993), where the distance is weighted by a positive definite matrix. The indirect inference of Gourieroux et al. (1993) has established conditions for consistent estimates, optimal weighting matrix for deviations between the auxiliary and simulated models, consistent estimators for the variance-covariance matrix and model specification tests. Similarly, the efficient method of moments established by Gallant and Tauchen (1996) would also be very useful. We would pursue this further to take full advantage of indirect inference and efficient method of moments in the future research 15

TABLE 4.2. The selected parameters of the MF models

\begin{tabular}{cccccccccc}
\hline$\alpha$ & $\gamma$ & $a_{1}$ & $a_{2}$ & $\mu$ & $m$ & $\delta$ & $b$ & $\sigma$ & $\sigma_{\delta}$ \\
\hline 0.858 & 8.464 & 6.024 & 0.383 & 0.946 & -0.200 & 0.292 & 6.763 & 0.24 & 3.473 \\
\hline
\end{tabular}

4.3. The Autocorrelation Patterns of the Selected MF Model. It is interesting to see whether our selected model is able to replicate the power-law behaviour of the DAX 30 described in Figure 4.2. Using the selected parameters in Table 4.2, we run 1,000 independent simulations for the MF model. For each run, we estimate the autocorrelation coefficients for returns, squared returns and absolute returns. We then take the average over the 1,000 runs and plot the ACs in Figure 4.3(a). From Figure 4.3(a), we see that for the MF model, the ACs are insignificant for the returns, but significantly positive over long lags for $r_{t}^{2}$ and $\left|r_{t}\right|$. Further, the sample autocorrelations for the absolute returns are greater than that for the squared returns at every

\footnotetext{
${ }^{14}$ For HAMs, model comparison have been discussed in Li et al. (2010) and Franke and Westhoff (2012). Franke and Westhoff (2012) suggest measures of model comparison if the models can be successfully estimated by the methods of simulated moments. Developing measures using (approximated) stochastic discount factor would provide better insight into HAMs, however, this seems not feasible for the paper at the moment. Behavioural finance literature often finds limits of arbitrage (see, e.g., Shleifer and Vishny, 1997; Froot and Dabora, 1999; Lamont and Thaler, 2003; and Gromb and Vayanos, 2010), verification of existence of stochastic discount factor is not trivial. We plan to explore it further in future research. We thank an anonymous referee to bring the issue of comparison of performance of HAMs to our attention.

${ }^{15}$ We would like to thank the referee for pointing this out.
} 


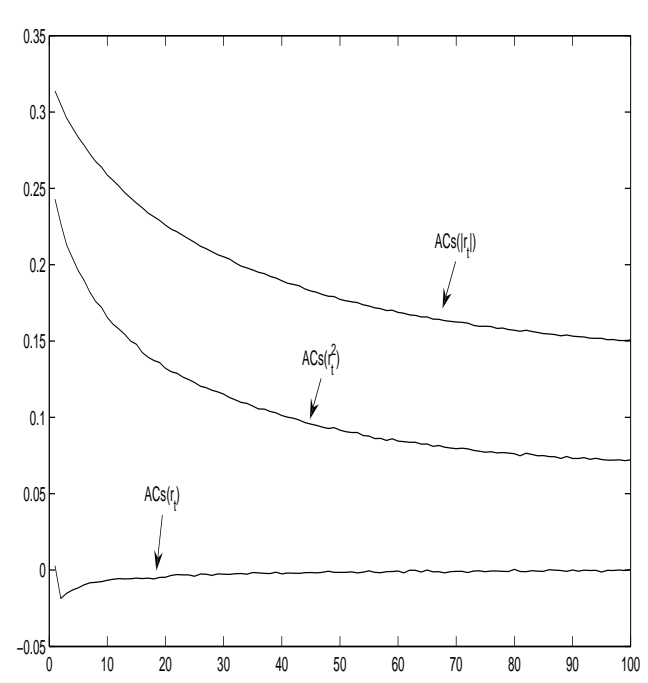

(a)
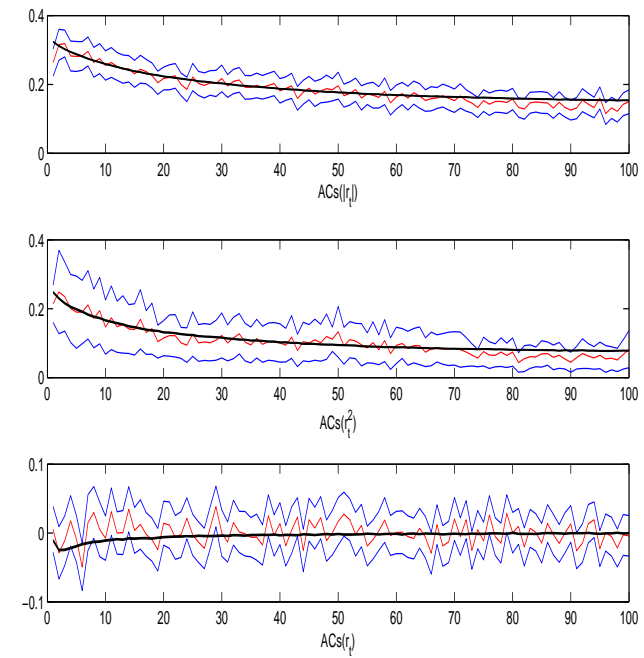

(b)

FIgURE 4.3. (a) Autocorrelations of $r_{t}, r_{t}^{2}$ and $\left|r_{t}\right|$ for the MF model. (b) The ACs of the returns, the squared returns and the absolute returns for the selected MF model and the DAX 30. The smooth lines refer to the MF model while the confidence intervals are those for the DAX 30.

lag up to at least 100 lags. Comparing to Figure 4.2 for the DAX 30, we see that they share the same patterns of decay of the autocorrelation functions of the return, squared return and absolute return. To see how well the selected model is able to match the autocorrelations of $r_{t}, r_{t}^{2}$ and $\left|r_{t}\right|$ for the DAX 30, in Figures 4.3(b), we plot the autocorrelation coefficients of returns, the squared returns and the absolute returns for the MF model together with the DAX 30 respectively. For comparison purposes, we use the Newey-West corrected standard errors and plot the corresponding confidence intervals of the ACs of the DAX 30. Figure 4.3 (b) clearly indicates that all of the autocorrelations of the MF model lie inside the confidence intervals of the DAX 30.

4.4. Estimates of Power-law Decay Index. Besides the visual inspection of autocorrelations of $r_{t}, r_{t}^{2}$ and $\left|r_{t}\right|$, one can also construct models to estimate the decay rate of the autocorrelations of $r_{t}, r_{t}^{2}$ and $\left|r_{t}\right|$. For instance, we can semiparametrically model long memory in a covariance stationary series $x_{t}, t=0, \pm 1, \ldots$, by

$$
s(\omega) \approx c_{1} \omega^{-2 d} \quad \text { as } \quad \omega \rightarrow 0^{+}
$$

where $0<c_{1}<\infty, s(\omega)$ is the spectral density of $x_{t}$, and $\omega$ is the frequency. Under (4.2), $s(\omega)$ has a pole at $\omega=0$ for $0<d<1 / 2$ (when there is a long memory in $x_{t}$ ). For $d \geq 1 / 2$, the 
process is not covariance stationary. For $d=0, s(\omega)$ is positive and finite. For $-1 / 2<d<0$, we have short memory, negative dependence, or antipersistence. The ACs can be described by $\rho_{k} \approx c_{2} k^{2 d-1}$, where $c_{2}$ is a constant and $\mu \equiv 2 d-1$ corresponds to the hyperbolic decay index.

Geweke and Poter-Hudak (1983), henceforth GPH, suggest a semiparametric estimator of the fractional differencing parameter $d$ based on a regression of the ordinates of the log spectral density. Given spectral ordinates $\omega_{j}=2 \pi j / T(j=1,2, \ldots, m)$, GPH suggest to estimate $d$ from

$$
\log I\left(\omega_{j}\right)=c-d \log \left(4 \sin ^{2}\left(\omega_{j} / 2\right)\right)+v_{j}
$$

where $v_{j}$ are assumed to be i.i.d. with zero mean and variance $\pi^{2} / 6$. If the number of ordinates $m$ is chosen such that $m=g(T)$ satisfying $\lim _{T \rightarrow \infty} g(T)=\infty, \lim _{T \rightarrow \infty} g(T) / T=0$ and $\lim _{T \rightarrow \infty}\left(\log (T)^{2}\right) / g(T)=0$, then the OLS estimator of $d$ based on (4.3) has the limiting distribution

$$
\sqrt{m}\left(\hat{d}_{G P H}-d\right) \stackrel{d}{\rightarrow} \mathcal{N}\left(0, \frac{\pi^{2}}{24}\right)
$$

Robinson (1995) provides a formal proof for $-1 / 2<d<1 / 2$, Velasco (1999) proves the consistency of $\hat{d}_{G P H}$ in the case $1 / 2 \leq d<1$ and its asymptotic normality in the case $1 / 2 \leq$ $d<3 / 4$. It is clear from this result that the GPH estimator is not $\sqrt{T}$-consistent and in fact converges at a slower rate.

Another most often used estimator of $d$ has been developed by Robinson and Henry (1999), henceforth RH; they suggest a semiparametric Gaussian estimate of the memory parameter $d$, by considering

$$
\hat{d}_{R H}=\arg \min _{d} R(d), \quad R(d)=\log \left\{\frac{1}{m} \sum_{j=1}^{m} \omega_{j}^{2 d} I\left(\omega_{j}\right)\right\}-2 \frac{d}{m} \sum_{j=1}^{m} \log \omega_{j},
$$

in which $m \in(0,[T / 2])$. They prove that, under some conditions (see Robinson and Henry (1999)),

$$
\sqrt{m}\left(\hat{d}_{R H}-d\right) \stackrel{d}{\rightarrow} \mathcal{N}\left(0, \frac{1}{4}\right)
$$

when $m<[T / 2]$ such that $1 / m+m / T \rightarrow 0$ as $T \rightarrow \infty$.

A major issue in the application of the GPH and the RH estimators is the choice of $m$, due to the fact that there is limited knowledge available concerning this issue, see Geweke (1998) for instance. Hence it is a wise precaution to report the estimated results for a range of bandwidths. In our study, for both the GPH and the RH estimates of $d$, we report the corresponding estimates 
for $m=50,100,150,200$ and 250, respectively. For instance, for the DAX 30, Table A.1 in Appendix A reports the GPH and the RH estimates of $d$ for the returns, squared returns, and absolute returns, respectively. In each panel of Table A.1, the first row reports the results from the GPH and the RH estimates with $m=50$, the second row reports the results of the GPH and the RH estimates with $m=100$, and so on. Table A.2 in Appendix A is arranged similarly.

For the DAX 30, we see from Table A.1 that the estimated $d$ for the returns are not significant at any conventional significance levels while those for the squared returns, and the absolute returns are significant. Thus the DAX 30 displays a clear evidence of power-law for the squared and the absolute returns where $d$ is positive, and the persistence in the absolute returns is much stronger than that in the squared returns. These results coincide with the well-established findings in the empirical finance literature.

For the selected MF model, the estimates of the decay rate $d$ are reported in Table A.2 in Appendix A, where the column 'Sig\%' indicates the percentage of simulations for which the corresponding estimates are significant at the 5\% level over 1,000 independent simulations. We find that on average the estimates of $d$ are insignificant for the returns, but significantly positive for the squared returns and the absolute returns. This verifies that there is a clear evidence of power-law for the squared returns and the absolute returns. It also shows that the patterns of the estimates of $d$ for the squared returns and the absolute returns are comparable to those of the DAX 30 in Table A.1.

The above analysis has clearly demonstrated that our selection procedure of model parameters is very effective in matching the autocorrelation patterns of the DAX 30. In the following discussion, we want to see if the selected MF model can be used to characterize the volatility clustering and power-law tail behaviour, for which our procedure is not designed.

4.5. Volatility Clustering, Power-law and (FI)GARCH Estimates. As a striking feature of the return series in financial markets, a number of econometric models of changing conditional variance have been developed to test and measure volatility clustering. The most widely used one is the one introduced by Engle (1982) and its generalization, the GARCH model, introduced by Bollerslev (1986). Following their specification if we model the returns as an AR (1) process, 
then a GARCH $(p, q)$ model is defined by:

$$
\left\{\begin{array}{l}
r_{t}=a+b r_{t-1}+\varepsilon_{t}, \quad \varepsilon_{t}=\sigma_{t} z_{t}, \\
\sigma_{t}^{2}=\alpha_{0}+\alpha(L) \varepsilon_{t}^{2}+\beta(L) \sigma_{t}^{2}, \quad z_{t} \sim N(0,1),
\end{array}\right.
$$

where $L$ is the lag operator, $\alpha(L)=\sum_{i=1}^{q} \alpha_{i} L^{i}$ and $\beta(L)=\sum_{j=1}^{p} \beta_{j} L^{j}$. Defining $v_{t}=\varepsilon_{t}^{2}-\sigma_{t}^{2}$, the process can be rewritten as an $\operatorname{ARMA}(s, p)$ process

$$
[1-\alpha(L)-\beta(L)] \varepsilon_{t}^{2}=\alpha_{0}+[1-\beta(L)] v_{t}
$$

with $s=\max \{p, q\}$. Table 4.3 reports the estimates of the GARCH $(1,1)$ model for the DAX 30, where the mean process follows an AR (1) structure. Based on the estimates, one can see that a small influence of the most recent innovation (small $\alpha_{1}$ ) is accompanied by a strong persistence of the variance coefficient (large $\beta_{1}$ ). It is also interesting to observe that the sum of the coefficients $\alpha_{1}+\beta_{1}$ is close to one, which indicates that the process is close to an integrated GARCH (IGARCH) process. Such parameter estimates are rather common when considering returns from high frequency daily financial data of both share and foreign exchange markets (see, Pagan (1996)). The GARCH implies that shocks to the conditional variance decay exponentially. However the IGARCH implies that the shocks to the conditional variance persist indefinitely.

TABLE 4.3. GARCH $(1,1)$ Estimates for the DAX 30

\begin{tabular}{ccccc}
\hline$a \times 10^{3}$ & $b$ & $\alpha_{0} \times 10^{4}$ & $\alpha_{1}$ & $\beta_{1}$ \\
\hline 0.4827 & 0.0539 & 0.0218 & 0.1056 & 0.8831 \\
$(0.1136)$ & $(0.0127)$ & $(0.0073)$ & $(0.0232)$ & $(0.0216)$ \\
\hline
\end{tabular}

Note: The numbers in parentheses are standard errors.

TABLE 4.4. FIGARCH $(1, d, 1)$ Estimates for the DAX 30

\begin{tabular}{cccccc}
\hline$a$ & $b$ & $\alpha_{0} \times 10^{4}$ & $d$ & $\phi_{1}$ & $\beta_{1}$ \\
\hline-0.0019 & 0.0012 & 0.0699 & 0.3259 & 0.2286 & 0.7716 \\
$(0.0003)$ & $(0.0092)$ & $(0.0248)$ & $(0.0078)$ & $(0.0148)$ & $(0.0034)$ \\
\hline
\end{tabular}

Note: The numbers in parentheses are standard errors.

In response to the finding that most of the financial time series are long memory volatility processes, Baillie et al. (1996) consider the Fractional Integrated GARCH (FIGARCH) process, where a shock to the conditional variance dies out at a slow hyperbolic rate. Chung (1999) 
suggests a slightly different parameterization of the model:

$$
\phi(L)(1-L)^{d}\left(\varepsilon_{t}^{2}-\sigma^{2}\right)=\alpha_{0}+[1-\beta(L)] v_{t},
$$

where $\phi(L)=1-\sum_{i=1}^{q} \phi_{i} L^{i}, \alpha_{0}=\phi(L)(1-L)^{d} \sigma^{2}$, and $\sigma^{2}$ is the unconditional variance of the corresponding GARCH model. Table 4.4 reports the estimates of the FIGARCH $(1, d, 1)$ model for the DAX 30, where the mean process follows an AR (1) model. The estimate for the fractional differencing parameter $\hat{d}$ is statistically very different from both zero and one. This is consistent with the well known finding that the shocks to the conditional variance die out at a slow hyperbolic rate.

TABLE 4.5. GARCH $(1,1)$ Estimates for the Selected MF Model

\begin{tabular}{ccccc}
\hline$a \times 10^{3}$ & $b$ & $\alpha_{0} \times 10^{4}$ & $\alpha_{1}$ & $\beta_{1}$ \\
\hline-0.0836 & 0.0241 & 0.3385 & 0.1009 & 0.9050 \\
$(0.6939)$ & $(0.0122)$ & $(0.1040)$ & $(0.0093)$ & $(0.0081)$ \\
\hline 1.0 & 53.2 & 85.9 & 99.9 & 100
\end{tabular}

Note: The numbers in parentheses are the standard errors, and the numbers in the last row are the percentages that the test statistics are significant at $5 \%$ level over 1000 independent simulations. This also holds for Table 4.6 .

TABLE 4.6. FIGARCH $(1, d, 1)$ Estimates for the Selected MF Model

\begin{tabular}{cccccc}
\hline$a$ & $b$ & $\alpha_{0} \times 10^{4}$ & $d$ & $\phi_{1}$ & $\beta_{1}$ \\
\hline-0.0381 & 0.0264 & 0.1116 & 0.4332 & 0.1922 & 0.7490 \\
$(0.1037)$ & $(0.0973)$ & $(0.2275)$ & $(0.0379)$ & $(0.0494)$ & $(0.0313)$ \\
\hline 74.4 & 66.3 & 4.3 & 89.2 & 89.2 & 95.2 \\
\hline
\end{tabular}

For the same specifications of the GARCH and FIGARCH models, we report resulting estimates for the selected MF model in Tables 4.5 and 4.6, respectively. Again, all these estimates are the average of the estimations for each independent run of the selected model. The results from the GARCH model are astonishingly similar to that from the DAX 30, that is, a small influence of the most recent innovation is accompanied by strong persistence of the variance coefficient and the sum of the coefficients $\alpha_{1}+\beta_{1}$ is close to one. For the estimates of the FIGARCH $(1, d, 1)$, we see that the estimate of $d$ for the selected MF model is significantly different from zero and one.

4.6. Power-law Tail Behaviour. Since the work of Mandelbrot (1963), power-law tail behaviour has been found in a wide range of financial time series, and it has become one of the 
salient features in financial markets 16 . In general, if $f_{\text {Normal }}$ is the probability density function of a normal distribution with mean $\mu$ and variance $\sigma^{2}$, then we have $\log f_{\text {Normal }}(x) \sim-\frac{1}{2 \sigma^{2}} x^{2}$ as $x \rightarrow \pm \infty$. A random variable $X$ is said to follow a power-law or Pareto distribution with shape parameter $\alpha>0$ and scale parameter $\beta>0$ if $\operatorname{Pr}[X>x]=(x / \beta)^{-\alpha}$, for $x \geq \beta$. In this case, $\log f_{\text {Pareto }}(x) \sim-(\alpha+1) \log (x)$ as $x \rightarrow+\infty$. Hence the difference of the tail behaviour between the normal and Pareto distribution is significant.

The estimation of tail index has been studied in great detail in Extreme Value Theory. More precisely, let $X_{1}, X_{2}, \ldots, X_{n}$ be a sequence of observations from some distribution function $F$, with its order statistics $X_{1, n} \leq X_{2, n} \leq \ldots \leq X_{n, n}$. As an analogue to the Central Limit Theorem, we know that, on average, if the maximum $X_{n, n}$, suitably centred and scaled, converges to a non-degenerate random variable, then there exist two sequences $\left\{a_{n}\right\}\left(a_{n}>0\right)$ and $\left\{b_{n}\right\}$ such that

$$
\lim _{n \rightarrow \infty} \operatorname{Pr}\left(\frac{X_{n, n}-b_{n}}{a_{n}} \leq x\right)=G_{\gamma}(x),
$$

where

$$
G_{\gamma}(x):=\exp \left(-(1+\gamma x)^{-1 / \gamma}\right)
$$

for some $\gamma \in R$ and $x$ such that $1+\gamma x>0$. Note that for $\gamma=0,-(1+\gamma x)^{-1 / \gamma}=e^{-x}$. If (4.10) holds, then we say that $F$ is in the max-domain of attraction of $G_{\gamma}$ and $\gamma$ is called the extreme value index. In Pareto distribution, the tail index $\gamma:=1 / \alpha$ measures the thickness of the tail distribution, the bigger the $\gamma$, the heavier the tail. The estimation of $\gamma$ has been thoroughly studied, see Beirlant et al. (2004) for a detailed account. We outline three major estimators, the Hill estimator, the Pickands estimator, and the moment estimator by Dekkers et al. (1989). The Hill index is defined by

$$
H_{k, n}=\frac{1}{k} \sum_{j=1}^{k} \log X_{n-j+1, n}-\log X_{n-k, n}
$$

This estimator is consistent for $k \rightarrow \infty, k / n \rightarrow 0$ as $n \rightarrow \infty$, and under extra conditions, $\sqrt{k}\left(H_{k, n}-\gamma\right)$ is asymptotically normal with mean 0 and variance $\gamma^{2}$. The Pickands estimator is defined as

$$
\hat{\gamma}_{P, k}=\frac{1}{\log 2} \log \left(\frac{X_{n-\lceil k / 4\rceil+1, n}-X_{n-\lceil k / 2\rceil+1, n}}{X_{n-\lceil k / 2\rceil+1, n}-X_{n-k+1, n}}\right) .
$$

\footnotetext{
${ }^{16}$ For a recent example, see the study by LeBaron and Samanta (2005) on international equity markets.
} 
The simplicity of the Pickands estimator is appealing but offset by large asymptotic variance, equal to $\gamma^{2}\left(2^{2 \gamma+1}+1\right)\left\{\left(2^{\gamma}-1\right) \log 2\right\}^{-2}$. Dekkers et al. (1989) introduce a moment estimator, which is a direct extension of Hill index,

$$
M_{k, n}=H_{k, n}+1-\frac{1}{2}\left(1-\frac{H_{k, n}^{2}}{H_{k, n}^{(2)}}\right)^{-1},
$$

where

$$
H_{k, n}^{(2)}=\frac{1}{k} \sum_{j=1}^{k}\left(\log X_{n-j+1, n}-\log X_{n-k, n}\right)^{2} .
$$

They also prove the consistency and asymptotic normality.

The Hill index relies on the average distance between extreme observations and the tail cutoff point to extrapolate the behaviour of the tails into the broader part of the distribution. In practice, the behaviour of the Hill index depends heavily on the choice of cutoff point $k$, which is also true for the other two estimators. This choice involves a tradeoff between bias and variance, which is well known in non-parametric econometrics. If $k$ is chosen conservatively with few order statistics in the tail, then the tail estimate will be sensitive to outliers in the distribution and have a high variance. On the other hand if the tail includes observations in the central part of the distribution, the variance is reduced but the estimate is biassed upward. So, we plot these estimates over a range of tail sizes. In the top panel of Figure 4.4, we plot the Hill index, we see that for the negative tail, the Hill index of the MF model fits in the confidence intervals of the DAX 30; for the positive tail, it fits well when $k$ is chosen less than 500. The Pickands estimates, plotted in the middle panel of Figurer 4.4, show a larger variability. It seems that on average the estimates from the MF model with selected parameters are not far away from those of the DAX 30. The moment estimates, plotted in the bottom panel of Figure 4.4 for the MF model are smaller than those for the DAX 30 but still fit in the confidence intervals. To conclude, the MF model exhibits power-law tail behaviour which is very close to that of the DAX 30.

The overall analysis in this section shows that the selection method of model parameter is very effective. The selected MF model is able to characterize successfully not only the powerlaw behaviour in autocorrelation, but also the volatility clustering and power-law tail behaviour in the DAX 30 as well. 

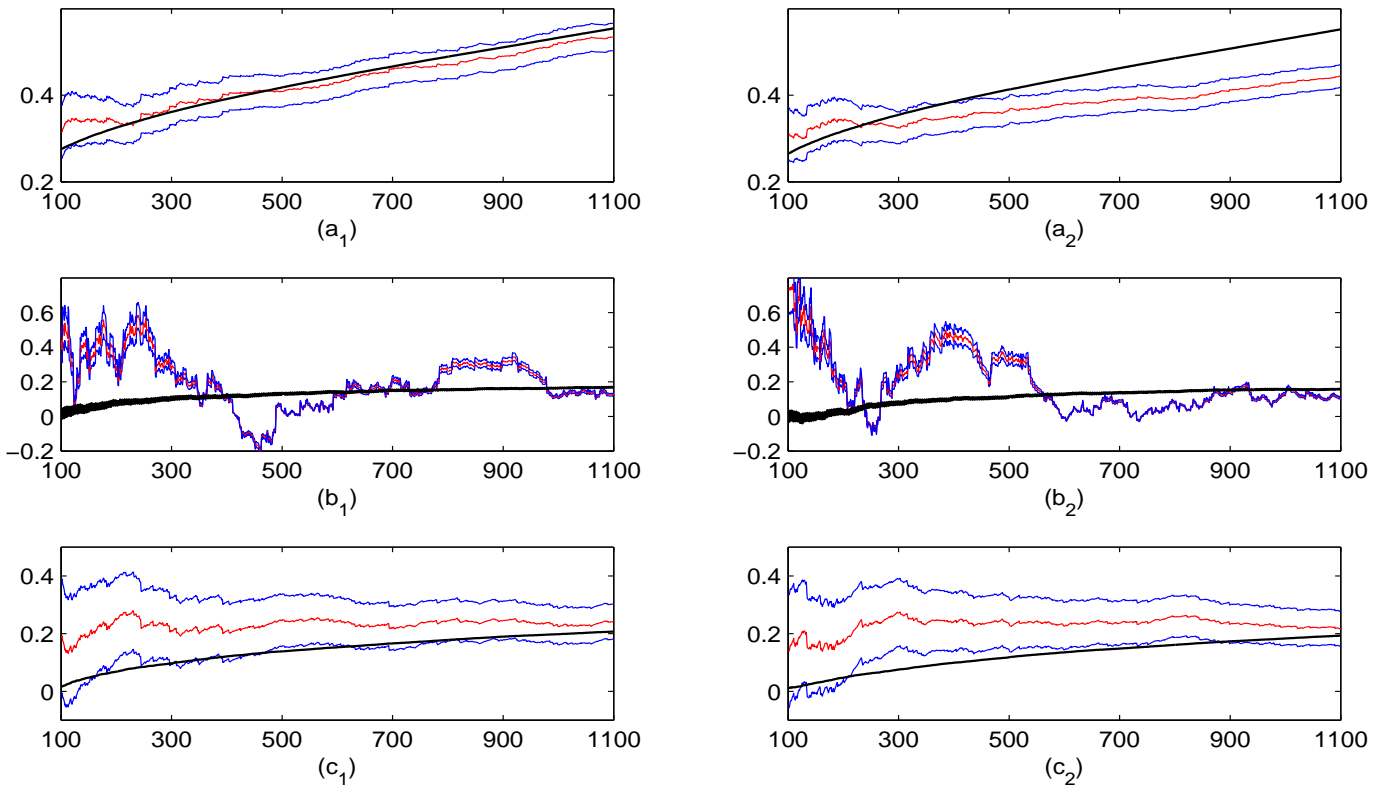

FIGURE 4.4. The tail index plots $\left(k, H_{k, n}\right),\left(k, \hat{\gamma}_{P, k}\right)$, and $\left(k, M_{k, n}\right)$ of the negative tails $\left(a_{1}\right),\left(b_{1}\right),\left(c_{1}\right)$ and the positive tails $\left(a_{2}\right),\left(b_{2}\right),\left(c_{2}\right)$ for the MF model and the DAX 30, respectively. The smooth lines refer to the MF model while the confidence intervals are those for the actual data.

\section{IMPLICATION AND EXPLANATION OF THE RESULT}

In this section, based upon the findings in $\mathrm{He}$ and $\mathrm{Li}(2007,2008)$ we aim to interpret the selected parameters and provide some implications and explanations on the generating mechanism of the power-law behaviour of the MF model.

For the model parameters in Table 4.2, the parameter $m=-0.2$ indicates that both the fundamentalists and trend followers are active in the market but the market is dominated by the trend followers with a majority of $60 \%$. The higher $a_{1}$ and lower $a_{2}$ imply that the fundamentalists are more risk averse when compared with the trend followers. Among the $40 \%$ of the fundamentalists, a high value of $\alpha=0.858$ indicates that their speed of price adjustment towards the fundamental value is high. A higher value of $\gamma=8.464$ indicates that trend followers extrapolate the price trend, measured by the difference between the current price and the geometric moving average of the history prices, strongly. The geometric decay rate is measured by $\delta$ and a value of $\delta=0.292$ gives a half life of 0.56 day, implying a very quick decaying weight. The parameter $b_{2}=b \sigma_{1}^{2}$ measures the influence of the sample variance $v_{t}$, in addition to the common belief on the price volatility $\sigma_{1}^{2}$, to the estimated price volatility for trend followers. The estimated value of $b=6.763$ implies that trend followers are cautious when estimating 
the price volatility, though they are less risk averse (measured by the CARA coefficient $a_{2}$ ). The estimated annual return volatility of $\sigma=24 \%$ is closer to the annual return volatility of $\sqrt{250} \times 0.01244=19.67 \%$ for the DAX 30. A value of $\mu=0.946$ indicates that the speed of the market price adjustment from the market maker is lower. The market price noise is about $3 \%$ of the average market price level. Intuitively, we have a very interesting market. The market is dominated by the trend followers. This dominance makes the market less stable. However, the market is balanced somehow by the activities of other market participants. More precisely, the trend followers destabilize the market in general. They are less risk averse, and extrapolate the price trend strongly. However, they are boundedly rational in a sense that they are cautious about their estimation of price volatility when they extrapolate and follow the price trend. The fundamentalists stabilize the market price to the fundamental value in general. They are more risk averse and adjust the market price to the fundamental value quickly. A low speed of price adjustment from the market maker can reduce the market impact of trend followers when the market becomes unstable. Therefore, due to trend following activity, the trend followers cause the market price to deviate from the fundamental value, but their cautiousness to high volatility (due to their own extrapolation, together with the activity of the fundamentalists), makes the market price move back to the fundamental value. The strength of the activity of the fundamentalists causes the market price to move back to the fundamental price very quickly, this trend in turn is extrapolated by the trend followers, pushing the market price to the other side of the fundamental value. This price process repeats again and again.

The above intuitive explanation based on the selected MF model can be verified by the dynamics of the underlying deterministic model, which has been examined extensively in $\mathrm{He}$ and Li (2008). In fact, given the selected parameters in the corresponding deterministic model, the constant fundamental equilibrium becomes unstable through a Hopf bifurcation, leading to (a)periodical oscillation of the market price around the fundamental equilibrium. Such periodical deviations of the market price from the fundamental value in the deterministic model are inherited by the stochastic model.

One of the important contributions of this paper is that the testing result provides strong support on the power-law behaviour mechanism examined in $\mathrm{He}$ and $\mathrm{Li}$ (2007). In $\mathrm{He}$ and Li (2007), the MF model is used to examine the potential source of agent-based models with heterogeneous belief in generating power-law behaviour in return autocorrelation patterns. By 
examining the dynamics of the underlying deterministic model and simulating the impact of two different forms of noisy process on the deterministic dynamics, $\mathrm{He}$ and $\mathrm{Li}$ (2007) find that the interaction of fundamentalists, risk-adjusted trend chasing from the trend followers and the interplay of noisy fundamental and demand processes and the underlying deterministic dynamics can be the source of power-law behaviour. In particular, it demonstrates that, for the MF model with a chosen set of parameters near the Hopf bifurcation value of the underlying deterministic model, adding noisy demand plays an important role in the generation of insignificant ACs on the returns, while the significant decaying AC patterns of the absolute returns and squared returns are more influenced by the noisy fundamental process. This potential source of power-law generating mechanism obtained in $\mathrm{He}$ and $\mathrm{Li}$ (2007) through experiment is verified from the systematic selection of model parameter we conduct in this paper. In particular, the selected parameters correspond to Hopf-bifurcation induced (a)periodic oscillation of the deterministic dynamics. Intuitively, the selected model in this paper should fit the data better than the experiment conducted in $\mathrm{He}$ and $\mathrm{Li}$ (2007) and this intuition is confirmed in the following discussion.

To see how well the MF model is able to describe the characteristics in the DAX 30, we construct confidence intervals for the estimates based upon the DAX 30 to see if the estimates based upon the selected MF model lie in these intervals or not. In the following, we focus on the average estimates of the MF model rather than their accuracy since, by running the MF model independently many times, the estimates converge much faster than those of the DAX 30. Apart from checking the confidence intervals, we also construct the Wald test for this purpose. For instance, for the decay index $d$ of the returns, the squared returns or the absolute returns, we want to test whether the values of the parameter $d$ estimated from both the DAX 30 and the MF model are the same. In other words, we want to test hypothesis:

$$
H_{0}: d_{D A X}=d_{M F}
$$

Using the Wald test, this null hypothesis can be tested by assuming that both the number of simulations and the number of time periods for each simulation go to infinity. In the construction of the Wald test, the test statistic is given by

$$
W=\left(\hat{d}_{D A X}-\hat{d}_{M F}\right) \hat{\Sigma}^{-1}\left(\hat{d}_{D A X}-\hat{d}_{M F}\right)
$$


where $\hat{\Sigma}$ is simply the variance of $\hat{d}_{D A X}$. The resulting test statistics are summarized in Table 5.1. In the column ' $r_{t}$ ', the first sub-row reports the test statistics corresponding to $\hat{d}_{G P H}$, and the second sub-row corresponding to $\hat{d}_{R H}$, and so on. Notice that the critical values of the Wald test at 5\% and $1 \%$ significant levels are 3.842 and 6.635, respectively. For the returns, we see that the estimated $d$ of the DAX 30 and the MF model are significantly different. However, for the squared returns and the absolute returns, the differences between the estimated $d$ of the DAX 30 and the MF model are not statistically significant. The same Wald test is also conducted for the simulation experiment in $\mathrm{He}$ and $\mathrm{Li}$ (2007) and the test statistics indicate that the estimated decay parameters from the estimation are much closer to the estimates from the DAX 30, when compared to the simulation experiment in $\mathrm{He}$ and $\mathrm{Li}$ (2007). For a more general discussion on the comparison of the simulation models with the real world data, see Li et al. $(2006,2010)$.

TABLE 5.1. The Wald test of $d$ with $m=50,100,150,200,250$

\begin{tabular}{c|ccccc}
\hline$m$ & 50 & 100 & 150 & 200 & 250 \\
\hline \multirow{2}{*}{$r_{t}$} & 18.58 & 45.71 & 64.69 & 71.22 & 84.57 \\
& 34.59 & 93.16 & 132.1 & 128.6 & 146.3 \\
\hline \multirow{2}{*}{$r_{t}^{2}$} & 0.044 & 1.263 & 0.303 & 0.015 & 0.055 \\
& 0.055 & 1.245 & 0.028 & 0.590 & 0.167 \\
\hline \multirow{2}{*}{$\left|r_{t}\right|$} & 0.078 & 1.097 & 1.668 & 0.484 & 0.266 \\
& 0.038 & 0.335 & 0.075 & 0.047 & 0.029 \\
\hline
\end{tabular}

The above analysis indicates that the selected market fraction model is able to replicate most of the stylized facts and power-law behaviour in the DAX 30, though formal statistical tests find that not all of the estimates from the selected MF model could easily match those of the DAX 30 simultaneously 13 . This is probably due to the simplicity of the MF model, but it is this simplicity that makes it possible to identify potential sources and mechanisms of the model in matching those characteristics in the DAX 30.

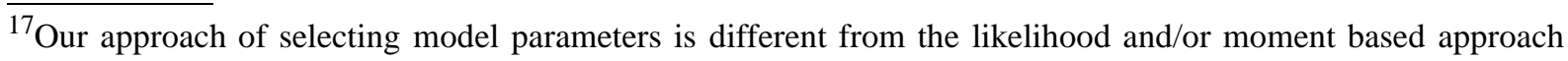
where the weighted average of the distance is minimized by taking into account the sampling error. As pointed out in Franke (2009), it is still possible to develop measures of goodness of fit. While the measures of goodness of fit are very useful when comparing the performance of different HAMs, the comparison results on various econometric characterizations between the MF model and the actual data seem to imply that it might be difficult to get meaningful test statistics. Thus, we have not explored this further. Moreover, in our approach the sampling error from the actual data is dealt with the confidence intervals of the estimates and that from the simulation data is eliminated by running many independent simulations.
} 


\section{CONCLUSION}

To characterize stylized facts and power-law behaviour in financial market, we have evidenced a growing literature in HAMs to incorporate agents' heterogeneity and bounded rationality. However, estimation of the HAMs to the power-law behaviour of financial data is a difficult and challenging task. This is due to heavy nonlinear nature and the wilderness of the HAMs that have too many parameters to be estimated, leading to less clear understanding of mechanisms that generate the stylized facts. This is also due to our limited understanding of the interplay between the deterministic dynamics and exogenous noise processes.

To understand these issues and to overcome the difficulty, He and Li (2008) develop a simple and parsimonious stochastic market fraction (MF) asset pricing model of two types of traders (fundamentalists and trend followers) under a market maker scenario. They seek to explain aspects of financial market behaviour, such as market dominance, convergence of the market price to the fundamental price, and under- and over-reaction. They also seek to characterize various statistical properties, including the convergence of the limiting distribution and autocorrelation structure of the stochastic model by using the dynamics of the underlying deterministic system, traders' heterogeneous behaviour and market fractions. The analysis underpins the mechanisms on various market behaviours (such as under/over-reactions), market dominance and stylized facts in high frequency financial markets. Based on these results, He and Li (2007) use the MF model to show that agent heterogeneity, risk-adjusted trend chasing through the geometric learning process, and the interplay of noisy fundamental and demand processes and the underlying deterministic dynamics can be the source of power-law distributed fluctuations. That analysis of the simple and parsimonious MF model provides a foundation for our testing in this paper.

Motivated by the mechanism investigation of the power-law properties of the simple and parsimonious MF model, this paper studies how to find a set of structural parameters of the model to match the power-law behaviour in the DAX 30. The selection method of model parameter is based on minimization of the average distance between the ACs of the returns, the squared returns and the absolute returns of the DAX 30 and the corresponding ACs generated from the MF model. With the parameter values of the selected model, we show that the ACs of the market fraction model share the same pattern as the DAX 30. To characterize the power-law 
behaviours statistically, we conduct econometric analysis via Monte Carlo simulations and estimate the decay indices, the (FI)GARCH parameters, Hill index and related tests. We find that the selected MF model matches closely to the corresponding estimates for the DAX 30 . We also demonstrate that the selected model parameters are consistent with the economic intuition of the model and support the generating mechanism of the power-law behaviour of the MF model found in $\mathrm{He}$ and $\mathrm{Li}$ (2007). As a by-product, the selected model also generates fat tails. Therefore the selected MF model can fit the most of the stylized facts observed in the DAX 30. Our results thus provide strong support for the explanatory power of the HAMs.

It is worth emphasizing that all these interesting qualitative and quantitative features arise from the simple model with fixed market fractions. It may be interesting to extend our analysis and to test the model established by Dieci et al. (2006), in which part of the market fractions is governed by market mood and the rest follows some adaptive switching process. One way to start might be to systematically select the model parameters first, and then implement misspecification tests. Allowing for market mood and switching mechanisms, we may gain a better characterization and understanding of the mechanisms driving financial markets and select the model to better fit the financial data. 


\section{ApPEndix A. Estimation Results}

TABLE A.1. The estimates of $d$ for the DAX 30 with $m=50,100,150,200,250$

\begin{tabular}{ccccc||cccc}
\hline & $\hat{d}_{G P H}$ & $t$ & $p$-value & $95 \%$ CI & $\hat{d}_{R H}$ & $t$ & $p$-value & $95 \%$ CI \\
\hline$r_{t}$ & 0.0014 & 0.014 & 0.989 & {$[-0.2005,0.2034]$} & -0.0179 & -0.253 & 0.801 & {$[-0.1565,0.1207]$} \\
& 0.0407 & 0.587 & 0.557 & {$[-0.0954,0.1769]$} & 0.0615 & 1.229 & 0.219 & {$[-0.0365,0.1595]$} \\
& 0.0548 & 0.985 & 0.325 & {$[-0.0542,0.1638]$} & 0.0829 & 2.031 & 0.042 & {$[0.0029,0.1629]$} \\
& 0.0406 & 0.852 & 0.394 & {$[-0.0528,0.1340]$} & 0.0482 & 1.362 & 0.173 & {$[-0.0211,0.1175]$} \\
& 0.0543 & 1.283 & 0.199 & {$[-0.0286,0.1372]$} & 0.0571 & 1.807 & 0.071 & {$[-0.0048,0.1191]$} \\
\hline$r_{t}^{2}$ & 0.4111 & 3.990 & 0.000 & {$[0.2091,0.6130]$} & 0.3785 & 5.353 & 0.000 & {$[0.2399,0.5171]$} \\
& 0.4527 & 6.518 & 0.000 & {$[0.3165,0.5888]$} & 0.4365 & 8.731 & 0.000 & {$[0.3385,0.5345]$} \\
& 0.4053 & 7.288 & 0.000 & {$[0.2963,0.5143]$} & 0.3735 & 9.149 & 0.000 & {$[0.2935,0.4535]$} \\
& 0.3666 & 7.696 & 0.000 & {$[0.2733,0.4600]$} & 0.3508 & 9.923 & 0.000 & {$[0.2816,0.4201]$} \\
& 0.3785 & 8.946 & 0.000 & {$[0.2956,0.4614]$} & 0.3605 & 11.40 & 0.000 & {$[0.2985,0.4225]$} \\
\hline$\left|r_{t}\right|$ & 0.5242 & 5.087 & 0.000 & {$[0.3222,0.7261]$} & 0.4801 & 6.790 & 0.000 & {$[0.3415,0.6187]$} \\
& 0.5495 & 7.911 & 0.000 & {$[0.4133,0.6856]$} & 0.5167 & 10.33 & 0.000 & {$[0.4187,0.6147]$} \\
& 0.5442 & 9.785 & 0.000 & {$[0.4352,0.6532]$} & 0.4914 & 12.04 & 0.000 & {$[0.4114,0.5714]$} \\
& 0.4993 & 10.48 & 0.000 & {$[0.4059,0.5927]$} & 0.4818 & 13.63 & 0.000 & {$[0.4125,0.5511]$} \\
& 0.4797 & 11.34 & 0.000 & {$[0.3968,0.5626]$} & 0.4708 & 14.89 & 0.000 & {$[0.4088,0.5327]$} \\
\hline
\end{tabular}

TABLE A.2. The estimates of $d$ for the MF model with $m=50,100,150,200,250$

\begin{tabular}{cccccc||ccccc}
\hline & $\hat{d}_{G P H}$ & $t$ & $p$-value & $95 \%$ CI & Sig\% & $\hat{d}_{R H}$ & $t$ & $p$-value & $95 \%$ CI & Sig\% \\
\hline$r_{t}$ & -0.4426 & -4.296 & 0.058 & {$[-0.4490,-0.4362]$} & 84.2 & -0.4337 & -6.134 & 0.039 & {$[-0.4381,-0.4294]$} & 91.0 \\
& -0.4292 & -6.179 & 0.034 & {$[-0.4335,-0.4249]$} & 91.9 & -0.4211 & -8.421 & 0.025 & {$[-0.4242,-0.4180]$} & 94.5 \\
& -0.3924 & -7.055 & 0.028 & {$[-0.3958,-0.3889]$} & 92.9 & -0.3860 & -9.454 & 0.019 & {$[-0.3885,-0.3834]$} & 95.4 \\
& -0.3611 & -7.580 & 0.023 & {$[-0.3641,-0.3582]$} & 94.2 & -0.3533 & -9.992 & 0.014 & {$[-0.3555,-0.3511]$} & 96.2 \\
& -0.3347 & -7.910 & 0.022 & {$[-0.3373,-0.3320]$} & 94.2 & -0.3251 & -10.28 & 0.015 & {$[-0.3271,-0.3232]$} & 96.0 \\
\hline$r_{t}^{2}$ & 0.3896 & 3.781 & 0.020 & {$[0.3832,0.3960]$} & 91.4 & 0.3950 & 5.587 & 0.002 & {$[0.3906,0.3994]$} & 99.1 \\
& 0.3746 & 5.394 & 0.001 & {$[0.3703,0.3789]$} & 99.3 & 0.3807 & 7.615 & 0.000 & {$[0.3776,0.3838]$} & 100 \\
& 0.3747 & 6.738 & 0.000 & {$[0.3713,0.3782]$} & 100 & 0.3803 & 9.315 & 0.000 & {$[0.3778,0.3828]$} & 100 \\
& 0.3725 & 7.819 & 0.000 & {$[0.3695,0.3754]$} & 100 & 0.3780 & 10.69 & 0.000 & {$[0.3758,0.3802]$} & 100 \\
& 0.3686 & 8.711 & 0.000 & {$[0.3659,0.3712]$} & 100 & 0.3734 & 11.81 & 0.000 & {$[0.3715,0.3754]$} & 100 \\
\hline$\left|r_{t}\right|$ & 0.4954 & 4.808 & 0.002 & {$[0.4890,0.5018]$} & 98.7 & 0.4938 & 6.983 & 0.000 & {$[0.4894,0.4981]$} & 99.8 \\
& 0.4767 & 6.863 & 0.000 & {$[0.4724,0.4810]$} & 100 & 0.4758 & 9.516 & 0.000 & {$[0.4727,0.4789]$} & 100 \\
& 0.4724 & 8.494 & 0.000 & {$[0.4690,0.4759]$} & 100 & 0.4720 & 11.56 & 0.000 & {$[0.4695,0.4746]$} & 100 \\
& 0.4662 & 9.787 & 0.000 & {$[0.4633,0.4692]$} & 100 & 0.4665 & 13.19 & 0.000 & {$[0.4643,0.4687]$} & 100 \\
& 0.4579 & 10.82 & 0.000 & {$[0.4553,0.4606]$} & 100 & 0.4587 & 14.51 & 0.000 & {$[0.4567,0.4606]$} & 100 \\
\hline
\end{tabular}




\section{REFERENCES}

Alfarano, S., Lux, T. and Wagner, F. (2005), 'Estimation of agent-based models: the case of an asymmetric herding model', Computational Economics 26, 19-49.

Amilon, H. (2008), 'Estimation of an adaptive stock market model with heterogeneous agents', Journal of Empirical Finance 15, 342-362.

Aoki, M. and Yoshikawa, H. (2002), 'Demand saturation/creation and economic growth', Journal of Economic Behavior and Organization 48, 127-154.

Baillie, R. T., Bollerslev, T. and Mikkelsen, H. (1996), 'Fractionally integrated generalized autoregressive conditional heteroskedasticity', Journal of Econometrics 74, 3-30.

Beirlant, J., Goegebeur, Y., Segers, J. and Teugels, J. (2004), Statistics of Extremes: Theory and Applications, John Wiley \& Sons Inc.

Black, F. and Scholes, M. (1973), 'The pricing of options and corporate liabilities', Journal of Political Economy 81, 637-659.

Bollerslev, T. (1986), 'Generalized autoregressive conditional heteroskedasticity', Journal of Econometrics 31, 307-327.

Bollerslev, T., Engle, R. F. and Nelson, D. B. (1986), Arch models, in R. F. Engle and D. McFadden, eds, 'Handbook of Econometrics', Vol. 4 of Handbook of Econometrics, Elsevier, chapter 49, pp. 2959-3038.

Boswijk, H., Hommes, C. and Manzan, S. (2007), 'Behavioral heterogeneity in stock prices', Journal of Economic Dynamics and Control 31, 1938-1970.

Brock, W. and Hommes, C. (1997), 'A rational route to randomness', Econometrica 65, 1059-1095.

Brock, W. and Hommes, C. (1998), 'Heterogeneous beliefs and routes to chaos in a simple asset pricing model', Journal of Economic Dynamics and Control 22, 1235-1274.

Canova, J. (1994), 'Statistical inference in calibrated models', Journal of Applied Econometrics 9, $123-144$.

Chen, S.-H., Chang, C.-L. and Du, Y.-R. (2012), 'Agent-based economic models and econometrics', Knowledge Engineering Review 27, 187-219.

Chen, S.-H., Lux, T. and Marchesi, M. (2001), 'Testing for non-linear structure in an artificial financial market', Journal of Economic Behavior and Organization 46, 327-342.

Chiarella, C., Dieci, R. and Gardini, L. (2002), 'Speculative behaviour and complex asset price dynamics: a global analysis', Journal of Economic Behavior and Organization 49, 173-197.

Chiarella, C., Dieci, R. and Gardini, L. (2005), 'The dynamic interaction of speculation and diversification', Applied Mathematical Finance 12(1), 17-52.

Chiarella, C., Dieci, R. and He, X. (2009), Heterogeneity, market mechanisms and asset price dynamics, in T. Hens and K. Schenk-Hoppe, eds, 'Handbook of Financial Markets: Dynamics and Evolution', North-Holland, Elsevier, pp. 277-344.

Chiarella, C. and He, X. (2002), 'Heterogeneous beliefs, risk and learning in a simple asset pricing model', Computational Economics 19, 95-132.

Chiarella, C. and He, X. (2003a), 'Dynamics of beliefs and learning under $\mathbf{a}_{l}$-processes - the heterogeneous case', Journal of Economic Dynamics and Control 27, 503-531.

Chiarella, C. and He, X. (2003b), 'Heterogeneous beliefs, risk and learning in a simple asset pricing model with a market maker', Macroeconomic Dynamics 7, 503-536.

Chiarella, C., He, X., and Hommes, C. (2006a), 'A dynamic analysis of technical trading rules in financial markets', Journal of Economic Dynamics and Control 30, 1729-1753.

Chiarella, C., He, X., and Hommes, C. (2006b), 'Moving average rules as a source of market instability', Physica A 370, 12-17.

Chiarella, C., He, X., Hung, H. and Zhu, P. (2006), 'An analysis of the cobweb model with boundedly rational heterogeneous producers', Journal of Economic Behavior and Organization 61, 750-768.

Chung, C.-F. (1999), Estimating the fractionally integrated garch model, discussion paper, National Taiwan University.

De Grauwe, P. and Grimaldi, M. (2003), Bubbling and crashing exchange rates, CESifo Working Paper Series 1045, CESifo Group Munich.

De Grauwe, P. and Grimaldi, M. (2006), 'Exchange rate puzzles: A tale of switching attractors', European Economic Review 50, 1-33.

Dekkers, A., Einmahl, J. and de Haan, L. (1989), 'A moment estimator for the index of an extreme-value distribution', Annals of Statistics 17, 1833-1855.

Diebold, F., L., O. and J., B. (1998), 'Dynamic equilibrium economies: A framework for comparing models and data', Review of Economic Studies 65, 433-452.

Dieci, R., Foroni, I., Gardini, L. and He, X. (2006), 'Market mood, adaptive beliefs and asset price dynamics', Chaos, Solitons and Fractals 29, 520-534. 
Ding, Z., Granger, C. and Engle, R. (1993), 'A long memory property of stock market returns and a new model', Journal of Empirical Finance 1, 83-106.

Donald, S. G., Imbens, G. W. and Newey, W. K. (2008), Choosing the number of moments in conditional moment restriction models, working paper, MIT.

Dridi, R., Guay, A. and Renault, E. (2007), 'Indirect inference and calibration of dynamic stochastic general equilibrium models', Journal of Economics 136, 397-430.

Dridi, R. and Renault, E. (2000), Semi-parametric indirect inference, EM, 392., Suntory and Toyota International Centres for Economics and Related Disciplines, London School of Economics and Political Science.

Engle, R. (1982), 'Autoregressive conditional heteroscedasticity with estimates of the variance of uk inflation', Econometrica 50, 987-1008.

Fama, E. F. (1976), Foundations of Finance, Basic Books: New York.

Farmer, J., Gillemot, L., Lillo, F., Mike, S. and Sen, A. (2004), 'What really causes large price changes', Quantitative Finance 4, 383-397.

Francq, C. and Zakoian, J.-M. (2000), 'Estimating weak garch representations', Econometric Theory 16(05), 692728.

Franke, R. (2009), 'Applying the method of simulated moments to estimate a small agent-based asset pricing model', Journal of Empirical Finance 16, 804-815.

Franke, R. (2010), 'On the specification of noise in two agent-based asset pricing models', Journal of Economic Dynamics and Control 34, 1140-1152.

Franke, R. and Westerhoff, F. (2012), 'Structural stochastic volatility in asset pricing dynamics: Estimation and model contest', Journal of Economic Dynamics and Control 36, 1193-1211.

Froot, K. A. and Dabora, E. M. (1999), 'How are stock prices affected by the location of trade?', Journal of Financial Economics 53(2), 189-216.

Gallant, A. R. and Tauchen, G. (1996), 'Which Moments to Match?', Econometric Theory 12(4), 657-681.

Gaunersdorfer, A. and Hommes, C. (2007), A nonlinear structural model for volatility clustering, in G. Teyssiere and A. Kirman, eds, 'Long Memory in Economics', Springer, pp. 265-288.

Geweke, J. (1998), 'Comment on real and spurious long memory properties of stock-market data (by lobato, i., and n. savin)', Journal of Business and Economic Statistics 16, 269-271.

Geweke, J. (2006), Computational experiments and reality, working paper, University of Iowa.

Geweke, J. and Poter-Hudak, S. (1983), 'The estimation and application of long memory time series models', Journal of Time Series Analysis 4, 221-238.

Giardina, I. and Bouchaud, J.-P. (2003), 'Bubbles, crashes and intermittency in agent based market models', Eur. Pys. J. B 31, 421-437.

Gilli, M. and Winker, P. (2003), 'A global optimization heuristic for estimating agent-based model', Computational Statistics and Data Analysis 42, 299-312.

Gourieroux, C., Monfort, A. and Renault, E. (1993), 'Indirect inference', Journal of Applied Econometrics 8, 85118.

Gromb, D. and Vayanos, D. (2010), 'Limits of arbitrage', Annual Review of Financial Economics 2, 251-275.

Hamilton, J. (1994), Time Series Analysis, Princeton University Press.

Hansen, L. and Heckman, J. (1996), 'The empirical foundations of calibration', Journal of Economic Prospectives 10, 87-104.

Hansen, L. P. and Jagannathan, R. (1997), 'Assessing specification errors in stochastic discount factor models', Journal of Finance 52(2), 557-90.

$\mathrm{He}, \mathrm{X}$. and Li, Y. (2007), 'Power law behaviour, heterogeneity, and trend chasing', Journal of Economic Dynamics and Control 31, 3396-3426.

He, X. and Li, Y. (2008), 'Heterogeneity, convergence and autocorrelations', Quantitative Finance 8, 58-79.

Hommes, C. (2001), 'Financial markets as nonlinear adaptive evolutionary systems', Quantitative Finance 1, 149_ 167.

Hommes, C. (2002), 'Modeling the stylized facts in finance through simple nonlinear adaptive systems', Proceedings of National Academy of Science of the United States of America 99, 7221-7228.

Hommes, C. H. (2006), Heterogeneous agent models in economics and finance, in L. Tesfatsion and K. L. Judd, eds, 'Handbook of Computational Economics', Vol. 2 of Handbook of Computational Economics, Elsevier, chapter 23, pp. 1109-1186.

Kan, R. and Robotti, C. (2008), 'Specification tests of asset pricing models using excess returns', Journal of Empirical Finance 15(5), 816-838.

Kan, R. and Robotti, C. (2009), 'Model comparison using the Hansen-Jagannathan distance', Review of Financial Studies 22(9), 3449-3490.

Kesten, H. (1973), 'Random difference equations and renewal theory for products of random matrices', Acta Mathematica 131, 207-248. 
Kirman, A. (1991), Epidemics of opinion and speculative bubbles in financial markets, in M. Taylor, ed., 'Money and Financial Markets', Blackwell, Cambridge, pp. 354-368.

Kirman, A. (1993), 'Ants, rationality, and recruitment', Quarterly Journal of Economics 108, 137-156.

Kydland, F. and Prescott, E. (1982), 'Time to build and aggregate fluctuations', Econometrica 50, 1345-1370.

Kydland, F. and Prescott, E. (1991), 'The econometrics of the general equilibrium approach to business cycles', Scandinavian Journal of Economics 93, 161-178.

Kydland, F. and Prescott, E. (1996), 'The computational experiment: an econometric tool', Journal of Economic Prospectives 10, 69-86.

Lamont, O. A. and Thaler, R. H. (2003), 'Can the market add and subtract? Mispricing in tech stock carve-outs', Journal of Political Economy 111(2), 227-268.

LeBaron, B. (2006), Agent-based computational finance, in L. Tesfatsion and K. L. Judd, eds, 'Handbook of Computational Economics', Vol. 2 of Handbook of Computational Economics, Elsevier, chapter 24, pp. 11871233.

LeBaron, B. and Samanta, R. (2005), Extreme value theory and fat tails in equity markets, working paper, Brandeis University.

Li, Y., Donkers, B. and Melenberg, B. (2006), The non- and semiparametric analysis of MS models: Some applications, CentER Discussion Paper 2006-95, Tilburg University.

Li, Y., Donkers, B. and Melenberg, B. (2010), 'Econometric analysis of microscopic simulation models', Quantitative Finance 10, 1187-1201.

Lintner, J. (1965), 'The valuation of risk assets and the selection of risky investments in stock portfolios and capital budgets', Review of Economics and Statistics 47, 13-37.

Lux, T. (1995), 'Herd behaviour, bubbles and crashes', Economic Journal 105, 881-896.

Lux, T. (1997), 'Time variation of second moments from a noise trader/infection model', Journal of Economic Dynamics and Control 22, 1-38.

Lux, T. (1998), 'The socio-economic dynamics of speculative markets: Interacting agents, chaos, and the fat tails of return distributions', Journal of Economic Behavior and Organization 33, 143-165.

Lux, T. (2008), Financial power laws: Empirical evidence, models and mechanisms, in C. Cioffi-Revilla, ed., 'Power Laws in the Social Sciences: Discovering Complexity and Non-Equilibrium in the Social Universe', Cambridge University Press.

Lux, T. (2009), Stochastic behavioural asset pricing and stylized facts, in T. Hens and K. Schenk-Hoppe, eds, 'Handbook of Financial Markets: Dynamics and Evolution', North-Holland, Elsevier, pp. 161-215.

Lux, T. and Marchesi, M. (1999), 'Scaling and criticality in a stochastic multi-agent model of a financial markets', Nature 397(11), 498-500.

Lux, T. and Schornstein, S. (2005), 'Genetic learning as an explanation of stylized facts of foreign exchange markets', Journal of Mathematical Economics 41, 169-196.

Lux, T. and Sornette, D. (2002), 'On rational bubbles and fat tails', Journal of Money, Credit and Banking 34(3), 589-610.

Mandelbrot, B. (1963), 'The variation of certain speculative prices', Journal of Business 36, 394-419.

Manzan, S. and Westerhoff, F. (2005), 'Representativeness of news and exchange rate dynamics', Journal of Economic Dynamics and Control 29, 677-689.

Markowitz, H. M. (1952), 'Portfolio selection', Journal of Finance 7(1), 77-91.

Mehra, R. and Prescott, E. (1985), 'The equity premium: a puzzle', Journal of Monetary Economics 15, 145-161.

Merton, R. (1971), 'Optimum consumption and portfolio rules in a continuous time model', Journal of Economic Theory 3, 373-413.

Merton, R. (1973a), 'An intertemporal capital asset pricing model', Econometrica 41, 867-887.

Merton, R. C. (1973b), ‘Theory of rational option pricing', Bell Journal of Economics 4(1), 141-183.

Mossin, J. (1966), 'Equilibrium in a capital asset market', Econometrica 35, 768-783.

Newey, W. K. and Windmeijer, F. (2009), 'Generalized method of moments with many weak moment conditions', Econometrica 77(3), 687-719.

Pagan, A. (1996), 'The econometrics of financial markets', Journal of Empirical Finance 3, 15-102.

Robinson, P. M. (1995), 'Log-periodgram regression of time series with long range dependence', Annals of Statistics 23, 1048-1072.

Robinson, P. M. and Henry, M. (1999), 'Long and short memory conditional heteroscedasticity in estimating the memory parameters of levels', Econometric Theory 15, 229-266.

Schorfheide, F. (2000), 'Loss function-based evaluation of DSGE models', Journal of Applied Econometrics 15, 645-670.

Sharpe, W. (1964), 'Capital asset prices: A theory of market equilibrium under conditions of risk', Journal of Finance 19, 425-442.

Shleifer, A. and Vishny, R. (1997), 'The limits of arbitrage', Journal of Finance 52, 35-55. 
Velasco, C. (1999), 'Non-stationary log-periodgram regression', Journal of Econometrics 91, 325-371.

Westerhoff, F. (2003), 'Speculative markets and the effectiveness of price limits', Journal of Economic Dynamics and Control 28, 439-508.

Westerhoff, F. (2004), 'Multiasset market dynamics', Macroeconomic Dynamics 8, 591-616.

Westerhoff, F. and Dieci, R. (2006), 'The effectiveness of keynes-tobin transaction taxes when heterogeneous agents can trade in different markets: a behavioral finance approach', Journal of Economic Dynamics and Control 30, 293-322.

Winker, P. and Gilli, M. (2003), Indirect estimation of the parameters of agent based models of financial markets, Technical report, University of Geneva. CEF2003 paper. 